\title{
ALTERNATING ARM EXPONENTS FOR THE CRITICAL PLANAR ISING MODEL ${ }^{1}$
}

\author{
BY HAO WU \\ Tsinghua University
}

\begin{abstract}
We derive the alternating arm exponents of the critical Ising model. We obtain six different patterns of alternating boundary arm exponents which correspond to the boundary conditions $(\ominus \oplus),(\ominus$ free) and (free free), and the alternating interior arm exponents.
\end{abstract}

1. Introduction. The Lenz-Ising model is one of the simplest models in statistical physics. It is a model on the spin configurations. Each vertex $x$ has a spin $\sigma_{x}$ which is $\oplus$ or $\ominus$. Each configuration of spins $\sigma=\left(\sigma_{x}, x \in V\right)$ has an intrinsic energy - the Hamiltonian: $H(\sigma)=-\sum_{x \sim y} \sigma_{x} \sigma_{y}$. A natural way to sample the random configuration is the Boltzman measure:

$$
\mu[\sigma] \propto \exp (-\beta H(\sigma)),
$$

where $\beta>0$ is the inverse-temperature. This measure favors configurations with low energy. Due to recent celebrated work of Chelkak and Smirnov [8, 9], it is proved that at the critical temperature, the interface of the Ising model is conformally invariant and converges to a random curve-Schramm-Loewner Evolution $\left(\mathrm{SLE}_{3}\right)$. In this paper, we drive the alternating arm exponents of the critical Ising model.

An arm is a simple path of $\oplus$ or of $\ominus$. We are interested in the decay of the probability that there are a certain number of arms of a certain pattern in the semiannulus $A^{+}(n, N)$ or annulus $A(n, N)$ connecting the inner boundary to the outer boundary. This probability should decay like a power in $N$ as $N \rightarrow \infty$, and the exponent in the power is called the critical arm exponents.

In $[15,16,22]$, the authors derived the value of the arm exponents for the critical percolation. As explained in [22], to derive the arm exponents, one needs three inputs: (1) the convergence of the interface to SLE; (2) the arm exponents of SLE; and (3) the quasi-multiplicativity. This strategy also works for the critical Ising model. In this paper, we derive the boundary arm exponents and the interior arm exponents of $\operatorname{SLE}_{\kappa}$ and its variant $\operatorname{SLE}_{\kappa}(\rho)$ (see Section 3.1 for the definition and see Figure 1 for the idea), and then explain how to apply these formulae to get the alternating arm exponents of the critical Ising model.

Received June 2016; revised June 2017.

${ }^{1}$ Supported by NCCR/SwissMAP, ERC AG COMPASP and the Swiss NSF.

MSC2010 subject classifications. Primary 60J67; secondary 60K35.

Key words and phrases. Schramm-Loewner evolution, critical planar Ising model, arm exponent. 


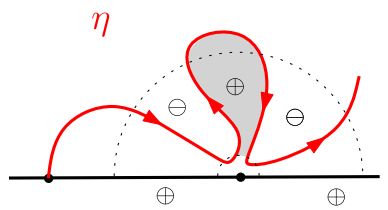

(a) $\alpha_{3}^{+}:(\ominus \ominus \ominus)$ with b.c. $(\oplus \oplus)$.

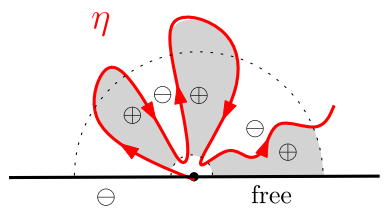

(d) $\beta_{5}^{+}:(\oplus \ominus \oplus \ominus \oplus)$ with b.c. $(\ominus$ free).

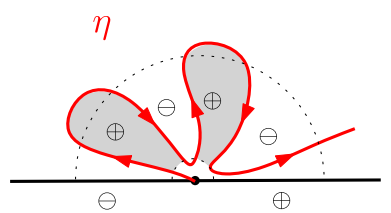

(b) $\alpha_{4}^{+}:(\oplus \ominus \oplus \ominus)$ with b.c. $(\ominus \oplus)$.

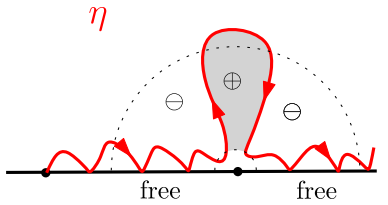

(e) $\gamma_{3}^{+}:(\ominus \ominus \ominus)$ with b.c. (free free).

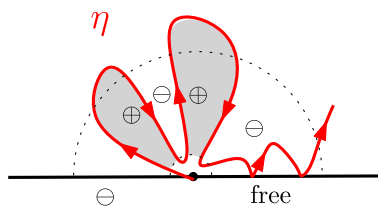

(c) $\beta_{4}^{+}:(\oplus \ominus \oplus \ominus)$ with b.c. ( $\ominus$ free).

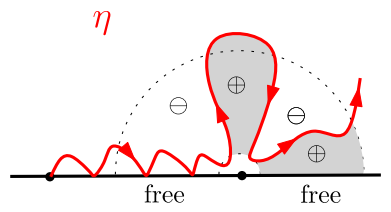

(f) $\gamma_{4}^{+}:(\ominus \oplus \ominus \oplus)$ with b.c. (free free).

FIG. 1. The six different patterns of boundary arm exponents in Theorem 1.1.

THEOREM 1.1. For the critical planar Ising model on the square lattice, we have the following six different patterns of the boundary arm exponents (the arm patterns are in clockwise order). Fix $j \geq 1$. We write b.c. for "boundary conditions."

- Consider the b.c. $(\oplus \oplus)$ and the pattern $(\ominus \oplus \ominus \cdots \oplus \ominus)$ with length $2 j-1$. The corresponding boundary arm exponents are given by

$$
\alpha_{2 j-1}^{+}=j(4 j+1) / 3 \text {. }
$$

- Consider the b.c. $(\ominus \oplus)$ and the pattern $(\oplus \ominus \cdots \oplus \ominus)$ with length $2 j$. The corresponding boundary arm exponents are given by

$$
\alpha_{2 j}^{+}=j(4 j+5) / 3 \text {. }
$$

- Consider the b.c. ( $\ominus$ free) and the pattern $(\oplus \ominus \oplus \cdots \ominus \oplus)$ with length $2 j-1$. The corresponding boundary arm exponents are given by

$$
\beta_{2 j-1}^{+}=2 j(2 j-1) / 3 \text {. }
$$

- Consider the b.c. ( $\ominus$ free) and the pattern $(\oplus \ominus \oplus \cdots \oplus \ominus)$ with length $2 j$. The corresponding boundary arm exponents are given by

$$
\beta_{2 j}^{+}=2 j(2 j+1) / 3 .
$$

- Consider the b.c. (free free) and the pattern $(\ominus \oplus \ominus \cdots \oplus \ominus)$ with length $2 j-1$. The corresponding boundary arm exponents are given by

$$
\gamma_{2 j-1}^{+}=(2 j-1)(4 j-3) / 6 \text {. }
$$


- Consider the b.c. (free free) and the pattern $(\ominus \oplus \cdots \ominus \oplus)$ with length $2 j$. The corresponding boundary arm exponents are given by

$$
\gamma_{2 j}^{+}=j(4 j-1) / 3 .
$$

THEOREM 1.2. For the critical planar Ising model on the square lattice, the alternating interior arm exponents with length $2 j$ for $j \geq 1$ are given by

$$
\alpha_{2 j}=\left(16 j^{2}-1\right) / 24 .
$$

REMARK 1.3. In Theorem 1.1, the arm exponent $\gamma_{2}^{+}=1$ is a universal arm exponent of the critical Ising model. In other words, the fact that $\gamma_{2}^{+}=1$ can be obtained by standard proof of universal arm exponents using RSW.

REMARK 1.4. For the critical planar Ising model (on the square lattice) in a topological rectangle $(\Omega ; a, b, c, d)$ with free boundary conditions, consider the probability that there exists a path of $\oplus$ connecting the boundary arc $(a b)$ to the boundary arc $(c d)$. It is proved in [5] that, as the mesh-size goes to zero, this probability converges to a function $f$ which maps topological rectangles to $[0,1]$ and it is conformally invariant. Therefore, the limit of this probability only depends on the extremal distance of the rectangle, whereas the exact formula for $f$ is unknown. As a consequence of Theorem 1.1, we can give the asymptotics of this function $f$. Consider the rectangle $[0, \pi L] \times[0,1]$ and let $f(L)$ be the limit of the probability that the Ising model with free boundary conditions has a $\oplus$ horizontal crossing of the rectangle. Then

$$
f(L)=\exp (-L(1 / 6+o(1))) .
$$

Relation to previous works. In this paper, we derive the arm exponents for $\operatorname{SLE}_{\kappa}(\rho)$ with $\kappa \in(0,4)$ and $\rho \in(-2,0]$. In [25], we derive the arm exponents of $\mathrm{SLE}_{\kappa}$ for $\kappa \in(4,8)$. The boundary 1 -arm exponent $\gamma_{1}^{+}$is related to the Hausdorff dimension of the intersection of $\operatorname{SLE}_{\kappa}(\rho)$ with the boundary which is $1-\gamma_{1}^{+}$. This dimension was obtained in [18, 24]. The formulae (1.1) and (1.2) are also obtained in [26]. The formulae (1.1) and (1.7) were predicted by KPZ in [11], equation (11.42), equation (11.43), and our work justifies those predictions.

The techniques developed in this paper are more complicated than those in [18, $22,25,26]$. One difficulty is that, when we estimate the arm events of $\operatorname{SLE}_{\kappa}(\rho)$ with $\kappa \in(0,4)$, we have two more variables to take care of than the cases in [22, 25, 26]; see the informal discussion at the end of Section 3.1. Another difficulty is that, when we derive the arm exponents by iteration, we do not allow error terms in the exponents, whereas, when one derives the one-point estimate for the intersection probability as in many other papers calculating the Hausdorff dimension of SLE curves, one is allowed to have error terms in the exponents (e.g., the conclusion as 
in Theorem A.1 is sufficient to derive the upper bound for the Hausdorff dimension). We treat these two difficulties in Section 3. There we obtain up-to-constant one-point estimates which guarantee the iteration.

Outline. We give preliminaries on SLE in Section 2. We derive the boundary arm exponents of $\operatorname{SLE}_{\kappa}(\rho)$ with $\kappa \in(0,4)$ and $\rho \in(-2,0]$ in Section 3. We derive the interior arm exponents of $\operatorname{SLE}_{\kappa}$ with $\kappa \in(0,4)$ in Section 4. Finally, we explain how to apply these formulae to obtain the alternating arm exponents of the critical Ising in Section 5 and complete the proof of Theorems 1.1 and 1.2.

2. Preliminaries on SLE. Notation. We denote by $f \lesssim g$ if $f / g$ is bounded from above by universal finite constant, by $f \gtrsim g$ if $f / g$ is bounded from below by a universal positive constant, and by $f \asymp g$ if $f \lesssim g$ and $f \gtrsim g$. We denote by

$$
f(\varepsilon)=g(\varepsilon)^{1+o(1)} \quad \text { if } \lim _{\varepsilon \rightarrow 0} \frac{\log f(\varepsilon)}{\log g(\varepsilon)}=1 .
$$

For $z \in \mathbb{C}, r>0$, we denote $B(z, r)=\{w \in \mathbb{C}:|w-z|<r\}$. We denote the unit $\operatorname{disc} B(0,1)$ by $\mathbb{U}$.

For two subsets $A, B \subset \mathbb{C}$, we $\operatorname{denote} \operatorname{dist}(A, B)=\inf \{|x-y|: x \in A, y \in B\}$. We assume that $\operatorname{dist}(A, \varnothing)=\infty$.

Let $\Omega$ be an open set and let $V_{1}, V_{2}$ be two sets such that $V_{1} \cap \bar{\Omega} \neq \varnothing$ and $V_{2} \cap$ $\bar{\Omega} \neq \varnothing$. We denote the extremal distance between $V_{1}$ and $V_{2}$ in $\Omega$ by $d_{\Omega}\left(V_{1}, V_{2}\right)$; see [2], Section 4, for the definition.

2.1. $\mathbb{H}$-hull and Loewner chain. We call a compact subset $K$ of $\overline{\mathbb{H}}$ an $\mathbb{H}$-hull if $\mathbb{H} \backslash K$ is simply connected. Riemann's mapping theorem and Schwarz' reflection principle assert that (see, e.g., [13], Proposition 3.34) there exists a unique conformal map $g_{K}$ from $\mathbb{H} \backslash K$ onto $\mathbb{H}$ such that $\lim _{|z| \rightarrow \infty}\left|g_{K}(z)-z\right|=0$. We call such $g_{K}$ the conformal map from $\mathbb{H} \backslash K$ onto $\mathbb{H}$ normalized at $\infty$.

The following Lemmas 2.1 to 2.3 are technical and they study the image of balls under conformal maps. They are crucial in the iteration when we derive the arm exponents in Section 3.

LEMMA 2.1. Let $K$ be an $\mathbb{H}$-hull and let $g_{K}$ be the conformal map from $\mathbb{H} \backslash K$ onto $\mathbb{H}$ normalized at $\infty$. Fix $x>0$ and $\varepsilon>0$ and assume that $x>\max (K \cap$ $\mathbb{R})$. Denote by $\gamma$ the connected component of $\mathbb{H} \cap(\partial B(x, \varepsilon) \backslash K)$ whose closure contains $x+\varepsilon$. Then $g_{K}(\gamma)$ is contained in the ball with center $g_{K}(x+\varepsilon)$ and radius $3\left(g_{K}(x+3 \varepsilon)-g_{K}(x+\varepsilon)\right)$, hence it is also contained in the ball with center $g_{K}(x+3 \varepsilon)$ and radius $8 \varepsilon g_{K}^{\prime}(x+3 \varepsilon)$.

ProOF. Define $r^{*}=\sup \left\{\left|z-g_{K}(x+\varepsilon)\right|: z \in g_{K}(\gamma)\right\}$. It suffices to show

$$
r^{*} \leq 3\left(g_{K}(x+3 \varepsilon)-g_{K}(x+\varepsilon)\right) .
$$


We will prove $(2.1)$ by estimating the extremal distance: $d_{\mathbb{H}}\left(g_{K}(\gamma),\left[g_{K}(x+\right.\right.$ $3 \varepsilon), \infty)$ ). By the conformal invariance and the comparison principle [2], Section 4.3, we can obtain the lower bound:

$$
\begin{aligned}
d_{\mathbb{H}}\left(g_{K}(\gamma),\left[g_{K}(x+3 \varepsilon), \infty\right)\right) & =d_{\mathbb{H} \backslash K}(\gamma,[x+3 \varepsilon, \infty)) \\
& \geq d_{\mathbb{H} \backslash B(x, \varepsilon)}(B(x, \varepsilon),[x+3 \varepsilon, \infty)) \\
& =d_{\mathbb{H} \backslash \mathbb{U}}(\mathbb{U},[3, \infty))=d_{\mathbb{H}}([-1,0],[1 / 3, \infty)) .
\end{aligned}
$$

In the last equality, we use the conformal map $\varphi(z):=(z+1 / z) / 4-1 / 2$ which sends $\mathbb{H} \backslash \mathbb{U}$ onto $\mathbb{H}$. Under this conformal map, we see that $\mathbb{U}$ is mapped to the interval $[-1,0]$ and $[3, \infty)$ is mapped to the interval $[1 / 3, \infty)$.

On the other hand, we will give an upper bound. Recall a fact for extremal distance: for $x<y$ and $r>0$, the extremal distance in $\mathbb{H}$ between $[y, \infty)$ and a connected set $S \subset \overline{\mathbb{H}}$ with $x \in \bar{S} \subset \overline{B(x, r)}$ is maximized when $S=[x-r, x]$; see [1], Chapter I-E, Chapter III-A. Since $g_{K}(\gamma)$ is connected and $g_{K}(x+\varepsilon) \in$ $\mathbb{R} \cap \overline{g_{K}(\gamma)}$, by the above fact, we have the upper bound:

$$
\begin{aligned}
& d_{\mathbb{H}}\left(g_{K}(\gamma),\left[g_{K}(x+3 \varepsilon), \infty\right)\right) \\
& \quad \leq d_{\mathbb{H}}\left(\left[g_{K}(x+\varepsilon)-r^{*}, g_{K}(x+\varepsilon)\right],\left[g_{K}(x+3 \varepsilon), \infty\right)\right) \\
& \quad=d_{\mathbb{H}}\left(\left[-r^{*}, 0\right],\left[g_{K}(x+3 \varepsilon)-g_{K}(x+\varepsilon), \infty\right)\right) .
\end{aligned}
$$

Combining the lower bound with the upper bound, we have

$$
d_{\mathbb{H}}([-1,0],[1 / 3, \infty)) \leq d_{\mathbb{H}}\left(\left[-r^{*}, 0\right],\left[g_{K}(x+3 \varepsilon)-g_{K}(x+\varepsilon), \infty\right)\right) .
$$

This implies (2.1) and completes the proof.

The following lemma is a standard estimate using the Koebe 1/4 theorem.

LEMMA 2.2. Fix $z \in \overline{\mathbb{H}}$ and $\varepsilon>0$. Let $K$ be an $\mathbb{H}$-hull and let $g_{K}$ be the conformal map from $\mathbb{H} \backslash K$ onto $\mathbb{H}$ normalized at $\infty$. Assume that $\operatorname{dist}(K, z) \geq 16 \varepsilon$. Then $g_{K}(B(z, \varepsilon))$ is contained in the ball with center $g_{K}(z)$ and radius $4 \varepsilon\left|g_{K}^{\prime}(z)\right|$.

Loewner chain is a collection of $\mathbb{H}$-hulls $\left(K_{t}, t \geq 0\right)$ associated with the family of conformal maps $\left(g_{t}, t \geq 0\right)$ obtained by solving the Loewner equation: for each $z \in \mathbb{H}$,

$$
\partial_{t} g_{t}(z)=\frac{2}{g_{t}(z)-W_{t}}, \quad g_{0}(z)=z
$$

where $\left(W_{t}, t \geq 0\right)$ is a one-dimensional real-valued continuous function which we call the driving function. Let $T_{z}$ be the swallowing time of $z$ defined as $\sup \{t \geq$ $\left.0: \min _{s \in[0, t]}\left|g_{s}(z)-W_{s}\right|>0\right\}$. Let $K_{t}:=\overline{\left\{z \in \mathbb{H}: T_{z} \leq t\right\}}$. Then $g_{t}$ is the unique conformal map from $H_{t}:=\mathbb{H} \backslash K_{t}$ onto $\mathbb{H}$ normalized at $\infty$. 
LEMMA 2.3. Suppose that $\left(K_{t}, t \geq 0\right)$ is a Loewner chain which is generated by a continuous curve $(\eta(t), t \geq 0)$. Fix $y \leq-4 r<0<x$. Let $\sigma$ be the first time that $\eta$ hits $B(y, r)$ and assume that $x$ is not swallowed by $\eta[0, \sigma]$ and that $y-r$ is not swallowed by $\eta[0, \sigma]$. Then we have

$$
g_{\sigma}(x)-W_{\sigma} \geq(x-y-2 r) / 2 .
$$

PROOF. Let $\gamma$ be the right-hand side of $\eta[0, \sigma]$. We prove the conclusion by estimating the extremal distance $d_{\mathbb{H} \backslash \eta[0, \sigma]}((-\infty, y-r), \gamma \cup[0, x])$. Denote $g_{\sigma}-$ $W_{\sigma}$ by $f$. On the one hand, by the conformal invariance of the extremal distance, we have

$$
\begin{aligned}
d_{\mathbb{H} \backslash \eta[0, \sigma]}((-\infty, y-r), \gamma \cup[0, x]) & =d_{\mathbb{H}}((-\infty, f(y-r)),(0, f(x))) \\
& =d_{\mathbb{H}}\left((-\infty, 0),\left(1, \frac{f(x)-f(y-r)}{-f(y-r)}\right)\right) .
\end{aligned}
$$

On the other hand, we give the following upper bound. Since any rectifiable arc in $\mathbb{H} \backslash B(y, r)$ connecting $(-\infty, y-r)$ and $(y+r, x)$ contains a rectifiable arc in $\mathbb{H} \backslash \eta[0, \sigma]$ connecting $(-\infty, y-r)$ and $\gamma \cup[0, x]$ [recall that $\sigma$ is the first time that $\eta$ hits $B(y, r)]$. By the comparison principle of the extremal distance [2], Section 4.3 , we have

$$
\begin{aligned}
d_{\mathbb{H} \backslash \eta[0, \sigma]}((-\infty, y-r), \gamma \cup[0, x]) \\
\quad \leq d_{\mathbb{H} \backslash B(y, r)}((-\infty, y-r),(y+r, x)) \\
\quad=d_{\mathbb{H}}\left((-\infty, 0),\left(1, \frac{1}{2}+\frac{x-y}{4 r}+\frac{r}{4(x-y)}\right)\right) .
\end{aligned}
$$

Comparing these two parts, we have

$$
\frac{f(x)-f(y-r)}{-f(y-r)} \geq \frac{1}{2}+\frac{x-y}{4 r}+\frac{r}{4(x-y)} \geq \frac{1}{2}+\frac{x-y}{4 r} .
$$

Thus

$$
\frac{g_{\sigma}(x)-W_{\sigma}}{W_{\sigma}-g_{\sigma}(y-r)} \geq \frac{x-y}{4 r}-\frac{1}{2} .
$$

Since the quantity $g_{t}(x)-g_{t}(y-r)$ is increasing in $t$, we have

$$
g_{\sigma}(x)-g_{\sigma}(y-r) \geq x-y+r .
$$

We denote $g_{\sigma}(x)-W_{\sigma}$ by $A$ and $W_{\sigma}-g_{\sigma}(y-r)$ by $B$. Then the above two estimates can be written as

$$
A \geq \frac{x-y-2 r}{4 r} B ; \quad A+B \geq x-y+r .
$$

As a consequence, we obtain

$$
A \geq \frac{x-y-2 r}{x-y+2 r}(A+B) \geq \frac{x-y-2 r}{x-y+2 r}(x-y+r) \geq(x-y-2 r) / 2 .
$$

This completes the proof. 
2.2. SLE processes. In this section, we introduce standard $\mathrm{SLE}_{\kappa}$ process, the process with one extra marked point $\operatorname{SLE}_{\kappa}(\rho)$ and the process with multiple marked points $\operatorname{SLE}_{\kappa}(\rho)$. The main statements in Sections 3 and 4 only concern $\operatorname{SLE}_{\kappa}(\rho)$ process; but, to derive those conclusions, one needs estimates for $\operatorname{SLE}_{\kappa}(\rho)$ process.

$\mathrm{SLE}_{\kappa}$ and $\operatorname{SLE}_{\kappa}(\rho)$ processes. An $\mathrm{SLE}_{\kappa}$ is the random Loewner chain $\left(K_{t}, t \geq 0\right)$ driven by $W_{t}=\sqrt{\kappa} B_{t}$ where $\left(B_{t}, t \geq 0\right)$ is a standard one-dimensional Brownian motion. In [20], the authors prove that $\left(K_{t}, t \geq 0\right)$ is almost surely generated by a continuous transient curve, that is, there almost surely exists a continuous curve $\eta$ such that for each $t \geq 0, H_{t}$ is the unbounded connected component of $\mathbb{H} \backslash \eta[0, t]$ and that $\lim _{t \rightarrow \infty}|\eta(t)|=\infty$.

For $\kappa>0$ and $\rho \in \mathbb{R}$, an $\operatorname{SLE}_{\kappa}(\rho)$ process is a Lowerner chain with one marked point $x \geq 0$. It is the Loewner chain driven by $W_{t}$ which is the solution to the system of SDEs:

$$
d W_{t}=\sqrt{\kappa} d B_{t}+\frac{\rho d t}{W_{t}-V_{t}}, \quad W_{0}=0 ; \quad d V_{t}=\frac{2 d t}{V_{t}-W_{t}}, \quad V_{0}=x .
$$

When $\rho>-2$, the process is well defined for all time and it is almost surely generated by a continuous transient curve. When $\rho \leq-2$, the process is well defined up to $T_{x}$ - the swallowing time of $x$. Moreover, the process is almost surely generated by a continuous curve up to and including $T_{x}$.

We summarize the behaviors of SLE for different $\rho$ 's in the following; see [10], Lemma 15. Fix $\kappa>0, \rho \in \mathbb{R}$ and $x>0$. Suppose that $\eta$ is an $\operatorname{SLE}_{\kappa}(\rho)$ process with force point $x$. The following facts hold almost surely:

- When $\rho \geq \kappa / 2-2$, the curve $\eta$ never hits the interval $(x, \infty)$.

- When $\kappa / 2-2>\rho>\kappa / 2-4$, the curve $\eta$ accumulates at a point in $(x, \infty)$ at finite time.

- When $\rho \leq \kappa / 2-4$, the curve $\eta$ converges to the point $x$ at finite time.

$\operatorname{SLE}_{\kappa}(\underline{\rho})$ processes. Next, we define an $\operatorname{SLE}{ }_{\kappa}\left(\underline{\rho}^{L} ; \underline{\rho}^{R}\right)$ process with multiple force points $\left(\underline{x}^{L} ; \underline{x}^{R}\right)$ where

$$
\begin{aligned}
& \underline{\rho}^{L}=\left(\rho^{l, L}, \ldots, \rho^{1, L}\right), \quad \underline{\rho}^{R}=\left(\rho^{1, R}, \ldots, \rho^{r, R}\right) \quad \text { with } \rho^{i, q} \in \mathbb{R} ; \\
& \underline{x}^{L}=\left(x^{l, L}<\cdots<x^{1, L} \leq 0\right), \quad \underline{x}^{R}=\left(0 \leq x^{1, R}<\cdots<x^{r, R}\right) .
\end{aligned}
$$

It is the Loewner chain driven by $W_{t}$ which is the solution to the following systems of SDEs:

$$
\begin{aligned}
d W_{t} & =\sqrt{\kappa} d B_{t}+\sum_{i} \frac{\rho^{i, L} d t}{W_{t}-V_{t}^{i, L}}+\sum_{i} \frac{\rho^{i, R} d t}{W_{t}-V_{t}^{i, R}}, \quad W_{0}=0 ; \\
d V_{t}^{i, L} & =\frac{2 d t}{V_{t}^{i, L}-W_{t}}, \quad V_{0}^{i, L}=x^{i, L}
\end{aligned}
$$




$$
d V_{t}^{i, R}=\frac{2 d t}{V_{t}^{i, R}-W_{t}}, \quad V_{0}^{i, R}=x^{i, R} .
$$

The solution exists and is unique up to the continuation threshold is hit-the first time $t$ that $W_{t}=V_{t}^{j, q}$ where $\sum_{1}^{j} \rho^{i, q} \leq-2$ for some $q \in\{L, R\}$. Moreover, the corresponding Loewner chain is almost surely generated by a continuous curve; see [17], Section 2 and Theorem 1.3.

In fact, in this paper, we only need the definitions and properties of SLE with three force points: $\operatorname{SLE}_{\kappa}\left(\rho^{L} ; \rho^{1, R}, \rho^{2, R}\right)$ with force points $\left(x^{L} ; x^{1, R}, x^{2, R}\right)$. To simplify notation, we will focus on these SLE processes in this section. From the Girsanov theorem, it follows that the law of an $\operatorname{SLE}_{\kappa}(\rho)$ process can be constructed by reweighting the law of an ordinary $\mathrm{SLE}_{\kappa}$ up to the first time that the Lowener chain swallows any force point [21], Theorem 6.

Lemma 2.4. Suppose $x^{L}<0<x^{1, R}<x^{2, R}$ and $\rho^{L}, \rho^{1, R}, \rho^{2, R} \in \mathbb{R}$. The following process is a local martingale for $\mathrm{SLE}_{\kappa}$ :

$$
\begin{aligned}
M_{t}= & g_{t}^{\prime}\left(x^{L}\right)^{\rho^{L}\left(\rho^{L}+4-\kappa\right) /(4 \kappa)}\left(g_{t}\left(x^{L}\right)-W_{t}\right)^{\rho^{L} / \kappa} \\
& \times g_{t}^{\prime}\left(x^{1, R}\right)^{\rho^{1, R}\left(\rho^{1, R}+4-\kappa\right) /(4 \kappa)}\left(g_{t}\left(x^{1, R}\right)-W_{t}\right)^{\rho^{1, R} / \kappa} \\
& \times g_{t}^{\prime}\left(x^{2, R}\right)^{\rho^{2, R}\left(\rho^{2, R}+4-\kappa\right) /(4 \kappa)}\left(g_{t}\left(x^{2, R}\right)-W_{t}\right)^{\rho^{2, R} / \kappa} \\
& \times\left(g_{t}\left(x^{1, R}\right)-g_{t}\left(x^{L}\right)\right)^{\rho^{L} \rho^{1, R} /(2 \kappa)}\left(g_{t}\left(x^{2, R}\right)-g_{t}\left(x^{L}\right)\right)^{\rho^{L} \rho^{2, R} /(2 \kappa)} \\
& \times\left(g_{t}\left(x^{2, R}\right)-g_{t}\left(x^{1, R}\right)\right)^{\rho^{1, R} \rho^{2, R} /(2 \kappa)} .
\end{aligned}
$$

The law of $\mathrm{SLE}_{\kappa}$ weighted by $M$ (up to the first time that $W$ hits one of the force points) is equal to the law of $\operatorname{SLE}_{\kappa}\left(\rho^{L} ; \rho^{1, R}, \rho^{2, R}\right)$ with force points $\left(x^{L} ; x^{1, R}, x^{2, R}\right)$.

The following two lemmas are technical estimates for SLE process with two marked points on the right. These two lemmas estimate the probability for SLE curves to have nice behavior and give lower bound for the probability uniform over the location of force points. We will use them in Section 3.

LEMMA 2.5. Fix $\kappa \in(0,4), \rho>-2, v \in \mathbb{R}$ that $\rho+v<\kappa / 2-4$. Suppose that $\eta$ is an $\operatorname{SLE}_{\kappa}(\rho, v)$ process with force points $(v, x)$ where $0 \leq v<x$. For $\varepsilon>0$, let $\tau$ be the first time that $\eta$ hits $B(x, \varepsilon)$. For $C \geq 4,1 / 4 \geq c>0$, define

$$
\mathcal{F}=\{\eta[0, \tau] \subset B(0, C x), \operatorname{dist}(\eta[0, \tau],[x-\varepsilon, C x]) \geq c \varepsilon\} .
$$

Then there exist constants $c, C, u_{0}>0$ which are uniform over $v, x, \varepsilon$ such that $\mathbb{P}[\mathcal{F}] \geq u_{0}$. 
PROOF. By the scale invariance of SLE, we may assume $x=1$. Let $\varphi(z)=$ $\varepsilon z /(1-z)$. Then $\varphi$ is the Möbius transformation of $\mathbb{H}$ that sends the triplet $(0,1, \infty)$ to $(0, \infty,-\varepsilon)$. Let us check the images of $\eta, B(0, C)$, and the $c \varepsilon$ neighborhood of $[x-\varepsilon, C \varepsilon]$ under $\varphi$, respectively.

Denote the image of $\eta$ under $\varphi$ by $\tilde{\eta}$, and denote its law by $\tilde{\mathbb{P}}$. Note that $\tilde{\eta}$ is an $\operatorname{SLE}_{\kappa}\left(\rho^{L} ; \rho^{R}\right)$ with force points $(-\varepsilon ; \varepsilon v /(1-v))$ where

$$
\rho^{L}=\kappa-6-\rho-v>\kappa / 2-2, \quad \rho^{R}=\rho>-2 .
$$

For $r \in(0,1 / 4)$ and $y \in(-1,0)$, let $\tilde{T}=\inf \{t: \tilde{\eta}(t) \in \partial B(y, r|y|)\}$ and $\tilde{S}=\inf \{t$ : $\tilde{\eta}(t) \in \partial B(0,1)\}$. Since $\rho^{L}>\kappa / 2-2$, by [18], Corollary 3.3, or Lemma A.3, there exists $A>1$ depending only on $\kappa, \rho^{L}, \rho^{R}$ such that

$$
\tilde{\mathbb{P}}[\tilde{T}<\tilde{S}, \operatorname{Im} \tilde{\eta}(\tilde{T}) \geq r|y| / 4] \leq r^{A} .
$$

Consider the image of $\mathbb{H} \backslash B(0, C)$ under $\varphi$. It is contained in the ball $B(-\varepsilon, 2 \varepsilon / C)$. By Lemma A.5, there exists a function $q(C)$, which depends on $C$ and is uniform over $\varepsilon$, such that the probability for $\tilde{\eta}$ to hit $B(-\varepsilon, 2 \varepsilon / C)$ is bounded by $q(C)$ and $q(C) \rightarrow 0$ as $C \rightarrow \infty$.

Consider the image of $c \varepsilon$-neighborhood of $[1+\varepsilon, C]$ under $\varphi$. Since $c \varepsilon$ neighborhood of $[1+\varepsilon, C]$ is contained in the union of the balls $B(1+k c \varepsilon / 4,4 c \varepsilon)$ for $4 / c \leq k \leq C / \varepsilon$, its image under $\varphi$ is contained in the union of the following balls:

$$
B\left(-4 /(c k)-\varepsilon, 256 /\left(c k^{2}\right)\right), \quad\lfloor 4 / c\rfloor \leq k \leq\lceil C / \varepsilon\rceil .
$$

Define $\tilde{\mathcal{F}}$ to be the event that $\tilde{\eta}$ exits the unit disc without touching the union of $B(-\varepsilon, 2 \varepsilon / C)$ and the image of $c \varepsilon$-neighborhood of $[1+\varepsilon, C]$ under $\varphi$. Then, by (2.3), we have

$$
1-\mathbb{P}[\mathcal{F}] \leq 1-\tilde{\mathbb{P}}[\tilde{\mathcal{F}}] \lesssim q(C)+\sum_{k=4 / c}^{C / \varepsilon}\left(\frac{1}{4 k+\varepsilon c k^{2}}\right)^{A} \lesssim q(C)+c^{A-1} .
$$

This implies the conclusion. In this proof, it is important that $A>1$ in (2.3). This explains the requirement $\rho+v<\kappa / 2-4$.

LEMMA 2.6. Fix $\kappa \in(0,4), \rho>-2, v \in \mathbb{R}$ that $\rho+v>-2$. Suppose that $\eta$ is an $\operatorname{SLE}_{\kappa}(\rho, v)$ process with force points $(v, x)$ where $0 \leq v \leq x$. For $r>0>y$ and $M>1$, assume $r<|y| \leq M r$. Let $\sigma$ be the first time that $\eta$ hits $B(y, r)$. For $C \geq 4,1 / 4 \geq c>0$, define

$$
\begin{aligned}
\mathcal{F}= & \{\sigma<\infty, \operatorname{dist}(\eta[0, \sigma], x) \geq c x, \eta[0, \sigma] \subset B(0, C|y|), \\
& \operatorname{dist}(\eta[0, \sigma],[C y, y]) \geq c r\} .
\end{aligned}
$$

Then there exist constants $c, C, u_{0}>0$ which may depend on $M$ but are uniform over $v, x, y, r$ such that $\mathbb{P}[\mathcal{F}] \geq u_{0}$. 
PROOF. From Lemma A.5, there exists a function $p(\delta)$ which is uniform over $v, x$ such that $p(\delta) \downarrow 0$ as $\delta \downarrow 0$ and that

$$
\mathbb{P}[\operatorname{dist}(\eta, x) \geq \delta x] \geq 1-p(\delta) .
$$

By scale invariance, we may assume $y=-1$ and $r \in[1 / M, 1)$. Next, we estimate the probability for the following event:

$$
\mathcal{G}=\{\sigma<\infty, \eta[0, \sigma] \subset B(0,4), \operatorname{dist}(\eta[0, \sigma],[-4,-1]) \geq r / 4\} .
$$

Denote by $f(v, x, r):=\mathbb{P}[\mathcal{G}]$. By a similar argument as in the proof of Lemma A.5, we see that $f$ is continuous and $q(M):=\inf f(v, x, r)>0$ where the infimum is over $0 \leq v \leq x$ and $r \in[1 / M, 1]$. Thus, $\mathbb{P}[\mathcal{G}] \geq q(M)$. Combining with (2.4), we have $\mathbb{P}[\mathcal{F}] \geq q(M)-p(c)$. This implies the conclusion.

\section{SLE boundary arm exponents.}

3.1. Definitions and statements. Fix $\kappa \in(0,4)$ and $\rho>-2$. Let $\eta$ be an $\operatorname{SLE}_{\kappa}(\rho)$ with force point $v \geq 0$. Assume $y \leq-4 r<0<\varepsilon \leq v \leq x$ and we consider the crossings of $\eta$ between $B(x, \varepsilon)$ and $B(y, r)$. Let $T_{x}$ be the first time that $\eta$ swallows $x$. We write c.c. for "connected component". We have four different types of crossing events.

Set $\tau_{0}=\sigma_{0}=0$. Let $\tau_{1}$ be the first time that $\eta$ hits $B(x, \varepsilon)$ and let $\sigma_{1}$ be the first time after $\tau_{1}$ that $\eta$ hits the c.c. of $\partial B(y, r) \backslash \eta\left[0, \tau_{1}\right]$ containing $y-r$. For $j \geq 1$, let $\tau_{j}$ be the first time after $\sigma_{j-1}$ that $\eta$ hits the c.c. of $\partial B(x, \varepsilon) \backslash \eta\left[0, \sigma_{j-1}\right]$ containing $x+\varepsilon$, and let $\sigma_{j}$ be the first time after $\tau_{j}$ that $\eta$ hits the c.c. of $\partial B(y, r) \backslash \eta\left[0, \tau_{j}\right]$ containing $y-r$. Define

$$
\mathcal{H}_{2 j-1}^{\alpha}(\varepsilon, x, y, r ; v)=\left\{\tau_{j}<T_{x}\right\}, \quad \mathcal{H}_{2 j}^{\beta}(\varepsilon, x, y, r ; v)=\left\{\sigma_{j}<T_{x}\right\} .
$$

In the definition of $\mathcal{H}_{2 j-1}^{\alpha}$ and $\mathcal{H}_{2 j}^{\beta}$, we are interested in the case when $x, y, r$ are fixed and $\varepsilon>0$ small. Imagine $\eta$ is the interface of the lattice model, then $\mathcal{H}_{2 j-1}^{\alpha}$ means that there are $2 j-1$ arms connecting $B(x, \varepsilon)$ to far away place; and $\mathcal{H}_{2 j}^{\beta}$ means that there are $2 j$ arms connecting $B(x, \varepsilon)$ to far away place.

Next, we define the other two types of crossing events. We emphasize that we will change the definition of the stopping times in the following. Set $\tau_{0}=\sigma_{0}=0$. Let $\sigma_{1}$ be the first time that $\eta$ hits $B(y, r)$ and $\tau_{1}$ be the first time after $\sigma_{1}$ that $\eta$ hits the c.c. of $\partial B(x, \varepsilon) \backslash \eta\left[0, \sigma_{1}\right]$ containing $x+\varepsilon$. For $j \geq 1$, let $\sigma_{j}$ be the first time after $\tau_{j-1}$ that $\eta$ hits the c.c. of $\partial B(y, r) \backslash \eta\left[0, \tau_{j-1}\right]$ containing $y-r$ and let $\tau_{j}$ be the first time after $\sigma_{j}$ that $\eta$ hits the c.c. of $\partial B(x, \varepsilon) \backslash \eta\left[0, \sigma_{j}\right]$ containing $x+\varepsilon$. Define

$$
\mathcal{H}_{2 j}^{\alpha}(\varepsilon, x, y, r ; v)=\left\{\tau_{j}<T_{x}\right\}, \quad \mathcal{H}_{2 j+1}^{\beta}(\varepsilon, x, y, r ; v)=\left\{\sigma_{j+1}<T_{x}\right\} .
$$

In the definition of $\mathcal{H}_{2 j}^{\alpha}$ and $\mathcal{H}_{2 j+1}^{\beta}$, we are interested in the case when $y, r$ are fixed and $x=\varepsilon>0$ small. Imagine $\eta$ is the interface of the lattice model, then 
$\mathcal{H}_{2 j}^{\alpha}$ means that there are $2 j$ arms connecting $B(x, \varepsilon)$ to far away place; and $\mathcal{H}_{2 j+1}^{\beta}$ means that there are $2 j+1$ arms connecting $B(x, \varepsilon)$ to far away place.

The definition here might be confusing at first sight, but these definitions avoid confusions in the proof. We emphasize that we define $\mathcal{H}_{n}^{\alpha}$ for odd $n$ in (3.1) and for even $n$ in (3.2); and that we define $\mathcal{H}_{n}^{\beta}$ for even $n$ in (3.1) and for odd $n$ in (3.2).

Propositions 3.1 and 3.2 study the probability of $\mathcal{H}_{n}^{\alpha}$ and $\mathcal{H}_{n}^{\beta}$ when the force point $v$ is close to $x$; Proposition 3.3 studies the probability of $\mathcal{H}_{n}^{\alpha}$ and $\mathcal{H}_{n}^{\beta}$ when the force point $v$ is far from $x$. Set $\alpha_{0}^{+}=0, \beta_{0}^{+}=0$ and $\gamma_{0}^{+}=0$. Assume $j \geq 1$.

Proposition 3.1. Fix $\kappa \in(0,4)$ and $\rho \in(-2,0]$. Define

$$
\alpha_{2 j-1}^{+}=2 j(2 j+\rho+2-\kappa / 2) / \kappa, \quad \alpha_{2 j}^{+}=2 j(2 j+\rho+4-\kappa / 2) / \kappa .
$$

Suppose $r \geq 1 \vee(200 \varepsilon)$. We have

$$
\begin{gathered}
\mathbb{P}\left[\mathcal{H}_{2 j-1}^{\alpha}(\varepsilon, x, y, r ; v)\right] \lesssim x^{\alpha_{2 j-2}^{+}-\alpha_{2 j-1}^{+}} \varepsilon^{\alpha_{2 j-1}^{+}} \\
\text {provided } 0 \leq x-v \lesssim \varepsilon, \text { and }|y| \geq(40)^{2 j-1} r \\
\mathbb{P}\left[\mathcal{H}_{2 j}^{\alpha}(\varepsilon, x, y, r ; v)\right] \lesssim x^{\alpha_{2 j}^{+}-\alpha_{2 j-1}^{+}} \varepsilon^{\alpha_{2 j-1}^{+}},
\end{gathered}
$$

$$
\text { provided } 0 \leq x-v \lesssim \varepsilon, \text { and }|y| \geq(40)^{2 j} r,
$$

where the constants in $\lesssim$ are uniform over $x$ and $\varepsilon$. We also have

$$
\mathbb{P}\left[\mathcal{H}_{2 j-1}^{\alpha}(\varepsilon, x, y, r ; v)\right] \gtrsim x^{\alpha_{2 j-2}^{+}-\alpha_{2 j-1}^{+}} \varepsilon^{\alpha_{2 j-1}^{+}},
$$

$$
\text { provided } 0 \leq x-v \lesssim \varepsilon \text {, and } x \asymp r \leq|y| \lesssim r,
$$

$$
\mathbb{P}\left[\mathcal{H}_{2 j}^{\alpha}(\varepsilon, x, y, r ; v)\right] \gtrsim x^{\alpha_{2 j}^{+}-\alpha_{2 j-1}^{+}} \varepsilon^{\alpha_{2 j-1}^{+}},
$$

$$
\text { provided } 0 \leq x-v \lesssim \varepsilon \text {, and } r \leq|y| \lesssim r,
$$

where the constants in $\gtrsim$ are uniform over $x$ and $\varepsilon$. In particular, we have

$$
\begin{gathered}
\mathbb{P}\left[\mathcal{H}_{2 j-1}^{\alpha}(\varepsilon, x, y, r ; v)\right] \asymp \varepsilon^{\alpha_{2 j-1}^{+}}, \\
\text {provided } 0 \leq x-v \lesssim \varepsilon, \text { and } \\
x \asymp r \leq(40)^{2 j-1} r \leq|y| \lesssim r, \\
\mathbb{P}\left[\mathcal{H}_{2 j}^{\alpha}(\varepsilon, x, y, r ; v)\right] \asymp \varepsilon^{\alpha_{2 j}^{+}}, \\
\text {provided } x \asymp v \asymp \varepsilon, \text { and }(40)^{2 j} r \leq|y| \lesssim r,
\end{gathered}
$$

where the constants in $\asymp$ are uniform over $\varepsilon$. 
Proposition 3.2. Fix $\kappa \in(0,4)$ and $\rho \in(-2, \kappa / 2-2)$. Define

$$
\beta_{2 j-1}^{+}=2 j(2 j+\kappa / 2-4-\rho) / \kappa, \quad \beta_{2 j}^{+}=2 j(2 j+\kappa / 2-2-\rho) / \kappa .
$$

Suppose $r \geq 1 \vee(200 \varepsilon)$. We have

$$
\mathbb{P}\left[\mathcal{H}_{2 j}^{\beta}(\varepsilon, x, y, r ; v)\right] \lesssim x^{\beta_{2 j-1}^{+}-\beta_{2 j}^{+}} \varepsilon^{\beta_{2 j}^{+}}
$$

$$
\text { provided } 0 \leq x-v \lesssim \varepsilon, \text { and }|y| \geq(40)^{2 j} r,
$$

$$
\mathbb{P}\left[\mathcal{H}_{2 j-1}^{\beta}(\varepsilon, x, y, r ; v)\right] \lesssim x^{\beta_{2 j-1}^{+}-\beta_{2 j-2}^{+}} \varepsilon^{\beta_{2 j-2}^{+}},
$$

$$
\text { provided } 0 \leq x-v \lesssim \varepsilon, \text { and }|y| \geq(40)^{2 j-1} r,
$$

where the constants in $\lesssim$ are uniform over $x$ and $\varepsilon$. We also have

$$
\mathbb{P}\left[\mathcal{H}_{2 j}^{\beta}(\varepsilon, x, y, r ; v)\right] \gtrsim x^{\beta_{2 j-1}^{+}-\beta_{2 j}^{+}} \varepsilon^{\beta_{2 j}^{+}},
$$

$$
\text { provided } 0 \leq x-v \lesssim \varepsilon, \text { and } x \asymp r \leq|y| \lesssim r,
$$$$
\mathbb{P}\left[\mathcal{H}_{2 j-1}^{\beta}(\varepsilon, x, y, r ; v)\right] \gtrsim x^{\beta_{2 j-1}^{+}-\beta_{2 j-2}^{+} \varepsilon^{\beta_{2 j-2}^{+}},}
$$

$$
\text { provided } 0 \leq x-v \lesssim \varepsilon, \text { and } r \leq|y| \lesssim r,
$$

where the constants in $\gtrsim$ are uniform over $x$ and $\varepsilon$. In particular, we have

$$
\begin{aligned}
\mathbb{P}\left[\mathcal{H}_{2 j}^{\beta}(\varepsilon, x, y, r ; v)\right] \asymp & \varepsilon^{\beta_{2 j}^{+},} \\
& \text {provided } 0 \leq x-v \lesssim \varepsilon, \text { and } x \asymp r \leq(40)^{2 j} r \leq|y| \lesssim r, \\
\mathbb{P}\left[\mathcal{H}_{2 j-1}^{\beta}(\varepsilon, x, y, r ; v)\right] \asymp \varepsilon^{\beta_{2 j-1}^{+},} & \text {provided } x \asymp v \asymp \varepsilon, \text { and }(40)^{2 j-1} r \leq|y| \lesssim r,
\end{aligned}
$$

where the constants in $\asymp$ are uniform over $\varepsilon$.

Proposition 3.3. Fix $\kappa \in(0,4)$ and $\rho \in(-2, \kappa / 2-2)$. Define

$$
\gamma_{2 j-1}^{+}=(2 j+\rho)(2 j+\rho+2-\kappa / 2) / \kappa, \quad \gamma_{2 j}^{+}=2 j(2 j+\kappa / 2-2) / \kappa .
$$

Define the event

$$
\mathcal{F}=\left\{\tau_{1}<T_{x}, \eta\left[0, \tau_{1}\right] \subset B(0, C x), \operatorname{dist}\left(\eta\left[0, \tau_{1}\right],[x-\varepsilon, x+3 \varepsilon]\right) \geq c \varepsilon\right\},
$$

where $c, C$ are the constants from Lemma 2.5. We have

$$
\mathbb{P}\left[\mathcal{H}_{2 j-1}^{\alpha}\left(\varepsilon, x, y, r ; 0^{+}\right) \cap \mathcal{F}\right] \asymp \varepsilon^{\gamma_{2 j-1}^{+}},
$$

$$
\text { provided } C x \leq r \leq(40)^{2 j-1} r \leq|y| \lesssim r,
$$




$$
\mathbb{P}\left[\mathcal{H}_{2 j}^{\beta}\left(\varepsilon, x, y, r ; 0^{+}\right) \cap \mathcal{F}\right] \asymp \varepsilon^{\gamma_{2 j}^{+}}
$$

$$
\text { provided } C x \leq r \leq(40)^{2 j} r \leq|y| \lesssim r,
$$

where the constants in $\asymp$ are uniform over $\varepsilon$.

We end this section by an informal discussion. Consider Proposition 3.1. Suppose we are allowed to ignore the evolution of the variables $y, r$ and $v$, and eliminate them from the notation. Then we can prove the conclusion by iteration. Let $\tau$ be the first time that $\eta$ hits the ball $B(x, \varepsilon)$, and denote $g_{\tau}-W_{\tau}$ by $f$. The image of the ball $B(x, \varepsilon)$ under $f$ is roughly a ball centered at $f(x)$ with radius $g_{\tau}^{\prime}(x) \varepsilon$. Thus we can write

$$
\mathbb{P}\left[\mathcal{H}_{2 j}^{\alpha}(\varepsilon, x)\right] \approx \mathbb{E}\left[\left(g_{\tau}^{\prime}(x) \varepsilon\right)^{\alpha_{2 j-1}^{+}} \mathbb{1}_{\{\tau<\infty\}}\right],
$$

and the conclusion can be deduced by an estimate on the expectation of $g_{\tau}^{\prime}(x)^{\lambda}$ for $\lambda \geq 0$. This is a natural first trial. However, the SLE curves can behave badly with small positive chance, and the evolution of the variables $y, r$ and $v$ can be arbitrary. In order to fulfill the above iteration procedure, we need to take care of all the variables. This explains the hard and lengthy work in Sections 3.2 and 3.3. In Section 3.2, we derive a more general version of the estimate on $\mathbb{E}\left[g_{\tau}^{\prime}(x)^{\lambda}\right]$. In Section 3.3, we prove Propositions 3.1 to 3.3 by iteration where the results in Section 3.2 play a crucial role.

3.2. Estimates on the derivatives. Suppose $\eta$ is an $\operatorname{SLE}_{\kappa}(\rho)$ with force point $v \geq 0$. We use the following notation: $g_{t}$ is the conformal map from the Loewner chain, $W_{t}$ is the driving function, $V_{t}$ is the evolution of the force point and $O_{t}$ is the rightmost point of $\eta[0, t] \cap \mathbb{R}$ under $g_{t}$.

LEMMA 3.4. Fix $\kappa \in(0,4)$ and $\rho>-2$. For $\lambda \geq 0$, define

$$
u_{1}(\lambda)=\frac{1}{\kappa}(\rho+4-\kappa / 2)+\frac{1}{\kappa} \sqrt{4 \kappa \lambda+(\rho+4-\kappa / 2)^{2}} .
$$

For $b \in \mathbb{R}$, assume

$$
4 b \geq(\lambda-b)(2 \rho+\kappa(\lambda-b)+4-\kappa) .
$$

Suppose $x \geq v>\varepsilon>0$ and let $\eta$ be an $\operatorname{SLE}_{\kappa}(\rho)$ with force point $v$. Define $\tau$ to be the first time that $\eta$ hits $B(x, \varepsilon)$ and $T$ to be the swallowing time of $x$. If $x=v$, we have

$$
\mathbb{E}\left[g_{\tau}^{\prime}(x)^{b}\left(g_{\tau}(x)-W_{\tau}\right)^{\lambda-b} \mathbb{1}_{\{\tau<T\}}\right] \asymp x^{-u_{1}(\lambda)} \varepsilon^{u_{1}(\lambda)+\lambda-b},
$$

where the constants in $\asymp$ are uniform over $x$ and $\varepsilon$. For $C \geq 4,1 / 4 \geq c>0$, define $\mathcal{F}=\{\tau<T, \operatorname{Im} \eta(\tau) \geq c \varepsilon, \eta[0, \tau] \subset B(0, C x), \operatorname{dist}(\eta[0, \tau],[-C x, y+r]) \geq c r\}$. 
There exist constants $C, c$ depending only on $\kappa$ and $\rho$ such that

$$
\begin{aligned}
\mathbb{E}\left[g_{\tau}^{\prime}(x)^{\lambda} \mathbb{1}_{\mathcal{F}}\right] \asymp x^{-u_{1}(\lambda)} \varepsilon^{u_{1}(\lambda)}, & \\
& \text { provided } 0 \leq x-v \lesssim \varepsilon, \text { and } x \asymp r \leq|y| \lesssim r,
\end{aligned}
$$

where the constants in $\asymp$ are uniform over $x$ and $\varepsilon$.

To prove Lemma 3.4, we only need to show the upper bound in (3.14) and the lower bound in (3.15). To show the upper bound in (3.14), we need the following Lemma 3.5 which is similar in the spirit of [23], Section 6.3; to show the lower bound in (3.15), we use Lemma 2.5.

LEMMA 3.5. Fix $\kappa>0$ and $v \leq \kappa / 2-4$. Let $\eta$ be an $\operatorname{SLE}_{\kappa}(v)$ with force point 1. Set $\Upsilon_{t}=\left(g_{t}(1)-O_{t}\right) / g_{t}^{\prime}(1), \sigma(s)=\inf \left\{t: \Upsilon_{t}=e^{-2 s}\right\}$, and $J_{t}=\left(V_{t}-\right.$ $\left.O_{t}\right) /\left(V_{t}-W_{t}\right)$. Let $T$ be the swallowing time of the point 1 . We have, for $\beta \geq 0$,

$$
\mathbb{E}\left[J_{\sigma(s)}^{-\beta} \mathbb{1}_{\{\sigma(s)<T\}} \asymp 1 \quad \text { when } 8+2 \nu+\kappa \beta<2 \kappa,\right.
$$

where the constants in $\asymp$ depend only on $\kappa, \nu, \beta$.

Proof. Since $0 \leq J_{t} \leq 1$, we only need to show the upper bound. Set $X_{t}=$ $V_{t}-W_{t}$. We know that

$$
d W_{t}=\sqrt{\kappa} d B_{t}+\frac{v d t}{W_{t}-V_{t}}, \quad d V_{t}=\frac{2 d t}{V_{t}-W_{t}} .
$$

By Itô's formula, we have

$$
d J_{t}=\frac{J_{t}}{X_{t}^{2}}\left(\kappa-v-2-\frac{2}{1-J_{t}}\right) d t+\frac{J_{t}}{X_{t}} \sqrt{\kappa} d B_{t}, \quad d \Upsilon_{t}=\Upsilon_{t} \frac{-2 J_{t} d t}{X_{t}^{2}\left(1-J_{t}\right)}
$$

Recall that $\sigma(s)=\inf \left\{t: \Upsilon_{t}=e^{-2 s}\right\}$, and denote by $\hat{X}, \hat{J}, \hat{\Upsilon}$ the processes indexed by $\sigma(s)$. Then we have

$$
\begin{aligned}
d \sigma(s) & =\hat{X}_{s}^{2} \frac{1-\hat{J}_{s}}{\hat{J}_{s}} d s, \\
d \hat{J}_{s} & =\left(\kappa-v-4-(\kappa-v-2) \hat{J}_{s}\right) d s+\sqrt{\kappa \hat{J}_{s}\left(1-\hat{J}_{s}\right)} d \hat{B}_{s},
\end{aligned}
$$

where $\hat{B}$ is a standard 1-dimensional Brownian motion. By [14], equations (56), (62), we know that $\hat{J}$ has an invariant density on $(0,1)$, which is proportional to $y^{1-(8+2 v) / \kappa}(1-y)^{4 / \kappa-1}$. Moreover, since $\hat{J}_{0}=1$, by a standard coupling argument, we may couple $\left(\hat{J}_{S}\right)$ with the stationary process $\left(\tilde{J}_{S}\right)$ that satisfies the same equation as $\left(\hat{J}_{s}\right)$, such that $\hat{J}_{s} \geq \tilde{J}_{s}$ for all $s \geq 0$. Then we get $\mathbb{E}\left[\hat{J}_{s}^{-\beta}\right] \leq \mathbb{E}\left[\tilde{J}_{s}^{-\beta}\right]$, which is a finite constant if $8+2 \nu+\kappa \beta<2 \kappa$. This gives the upper bound in (3.16) and completes the proof. 
Proof OF LemMA 3.4-(3.14). Define $\Upsilon_{t}=\left(g_{t}(x)-O_{t}\right) / g_{t}^{\prime}(x), J_{t}=$ $\left(g_{t}(x)-O_{t}\right) /\left(g_{t}(x)-W_{t}\right)$ and $\hat{\tau}_{\varepsilon}=\inf \left\{t: \Upsilon_{t}=\varepsilon\right\}$. Set

$$
M_{t}=g_{t}^{\prime}(x)^{(\nu-\rho)(\nu+\rho+4-\kappa) /(4 \kappa)}\left(g_{t}(x)-W_{t}\right)^{(\nu-\rho) / \kappa},
$$

where $v=\kappa / 2-4-\sqrt{4 \kappa \lambda+(\rho+4-\kappa / 2)^{2}}$. Then $M$ is a local martingale and the law of $\eta$ weighted by $M$ becomes the law of $\operatorname{SLE}_{\kappa}(v)$ with force point $x$ (see Lemma 2.4). Set $\beta=u_{1}(\lambda)+\lambda-b$. By the choice of $\nu$, we can rewrite

$$
M_{t}=g_{t}^{\prime}(x)^{b}\left(g_{t}(x)-W_{t}\right)^{\lambda-b} \Upsilon_{t}^{-\beta} J_{t}^{\beta} .
$$

At time $\hat{\tau}_{\varepsilon}<\infty$, we have $\Upsilon_{\hat{\tau}_{\varepsilon}}=\varepsilon$. Thus

$$
\begin{aligned}
& \mathbb{E}\left[g_{\hat{\tau}_{\varepsilon}}^{\prime}(x)^{b}\left(g_{\hat{\tau}_{\varepsilon}}(x)-W_{\hat{\tau}_{\varepsilon}}\right)^{\lambda-b} \mathbb{1}_{\left\{\hat{\tau}_{\varepsilon}<T\right\}}\right] \\
& \quad=\varepsilon^{\beta} M_{0} \mathbb{E}^{*}\left[\left(J_{\hat{\tau}_{\varepsilon}^{*}}^{*}\right)^{-\beta}\right]=\varepsilon^{\beta} x^{-u_{1}(\lambda)} \mathbb{E}^{*}\left[\left(J_{\hat{\tau}_{\varepsilon}^{*}}^{*}\right)^{-\beta}\right] \asymp \varepsilon^{\beta} x^{-u_{1}(\lambda)},
\end{aligned}
$$

where $\mathbb{P}^{*}$ is the law of $\eta$ weighted by $M$ and $\hat{\tau}_{\varepsilon}^{*}, J^{*}$ are defined accordingly. The last relation is due to Lemma 3.5. Thus we have

$$
\mathbb{E}\left[g_{\hat{\tau}_{\varepsilon}}^{\prime}(x)^{b}\left(g_{\hat{\tau}_{\varepsilon}}(x)-W_{\hat{\tau}_{\varepsilon}}\right)^{\lambda-b} \mathbb{1}_{\left\{\hat{\tau}_{\varepsilon}<T\right\}}\right] \asymp x^{-u_{1}(\lambda)} \varepsilon^{u_{1}(\lambda)+\lambda-b} .
$$

Consider the process $\left(U_{t}:=g_{t}^{\prime}(x)^{b}\left(g_{t}(x)-W_{t}\right)^{\lambda-b}\right)_{t \geq 0}$. We can check that it is a super martingale by Itô's formula when (3.13) holds. Combining with the fact $\hat{\tau}_{\varepsilon / 4} \geq \tau \geq \hat{\tau}_{4 \varepsilon}$, we have

$$
\mathbb{E}\left[U_{\hat{\tau}_{\varepsilon / 4}} \mathbb{1}_{\left\{\hat{\tau}_{\varepsilon / 4}<T\right\}}\right] \leq \mathbb{E}\left[U_{\tau} \mathbb{1}_{\{\tau<T\}}\right] \leq \mathbb{E}\left[U_{\hat{\tau}_{4 \varepsilon}} \mathbb{1}_{\left\{\hat{\tau}_{4 \varepsilon}<T\right\}}\right]
$$

Combining with (3.17), we obtain (3.14).

Proof of Lemma 3.4-(3.15). We may assume $x>v$. Define

$$
M_{t}=g_{t}^{\prime}(x)^{v(v+4-\kappa) /(4 \kappa)}\left(g_{t}(x)-W_{t}\right)^{v / \kappa}\left(g_{t}(x)-g_{t}(v)\right)^{\nu \rho /(2 \kappa)},
$$

where $v=-\kappa u_{1}(\lambda)$. Then $M$ is a local martingale for $\eta$ and the law of $\eta$ weighted by $M$ is an $\operatorname{SLE}_{\kappa}(\rho, v)$ with force points $(v, x)$ (see Lemma 2.4). We argue that

$$
g_{\tau}(x)-g_{\tau}(v) \asymp(x-v) g_{\tau}^{\prime}(x) .
$$

There are two possibilities: $v$ is swallowed by $\eta[0, \tau]$ or not. If $v$ is not swallowed by $\eta[0, \tau]$, then by the Koebe $1 / 4$ theorem, we know that $g_{\tau}(x)-g_{\tau}(v) \asymp(x-$ $v) g_{\tau}^{\prime}(x)$. If $v$ is swallowed by $\eta[0, \tau]$, then we must have $x-v \geq \varepsilon$. By the Koebe $1 / 4$ theorem, we have $g_{\tau}(x)-g_{\tau}(v) \asymp \varepsilon g_{\tau}^{\prime}(x)$. Since $\varepsilon \leq x-v \lesssim \varepsilon$, we have $g_{\tau}(x)-g_{\tau}(v) \asymp(x-v) g_{\tau}^{\prime}(x)$. These complete the proof of (3.18).

On $\{\operatorname{Im} \eta(\tau) \geq c \varepsilon\}$, we also have $g_{\tau}(x)-W_{\tau} \asymp \varepsilon g_{\tau}^{\prime}(x)$. Combining with (3.18) and the choice of $v$, we have

$$
M_{\tau} \asymp \varepsilon^{\nu / \kappa}(x-v)^{v \rho /(2 \kappa)} g_{\tau}^{\prime}(x)^{\lambda} \quad \text { on } \mathcal{F} \text {. }
$$


Therefore,

$$
\mathbb{E}\left[g_{\tau}^{\prime}(x)^{\lambda} \mathbb{1}_{\mathcal{F}}\right] \asymp \varepsilon^{-v / \kappa}(x-v)^{-v \rho /(2 \kappa)} M_{0} \mathbb{P}^{*}\left[\mathcal{F}^{*}\right]=\varepsilon^{u_{1}(\lambda)} x^{-u_{1}(\lambda)} \mathbb{P}^{*}\left[\mathcal{F}^{*}\right],
$$

where $\mathbb{P}^{*}$ is the law of $\eta$ weighted by $M$ and $\mathcal{F}^{*}$ is defined accordingly. Note that $\rho>-2, \rho+v<\kappa / 2-4$. By a similar proof of Lemma 2.5, we know that there exists constants $C, c$ such that $\mathbb{P}^{*}\left[\mathcal{F}^{*}\right] \asymp 1$. This completes the proof.

REMARK 3.6. Taking $\lambda=b=0$ in Lemma 3.4, we see Proposition 3.1 holds for $\mathcal{H}_{1}^{\alpha}$ with $\alpha_{1}^{+}=u_{1}(0)=2(\rho+4-\kappa / 2) / \kappa$. Precisely, taking $\lambda=0$ in (3.15), we have $\mathbb{P}[\eta$ hits $B(x, \varepsilon)] \gtrsim(\varepsilon / x)^{u_{1}(0)}$. This gives the lower bound. Taking $\lambda=b=0$ in (3.14), we have $\mathbb{P}[\eta$ hits $B(v, \varepsilon)] \asymp(\varepsilon / v)^{u_{1}(0)}$. Since $0 \leq x-v \lesssim \varepsilon$, we know that $B(x, \varepsilon)$ is contained in $B(v, \tilde{C} \varepsilon)$ for some constant $\tilde{C}$, thus

$$
\mathbb{P}[\eta \text { hits } B(x, \varepsilon)] \leq \mathbb{P}[\eta \text { hits } B(v, \tilde{C} \varepsilon)] \asymp(\varepsilon / v)^{u_{1}(0)} \lesssim(\varepsilon / x)^{u_{1}(0)} .
$$

This gives the upper bound.

LEMma 3.7. Fix $\kappa \in(0,4)$ and $\rho \in(-2,0]$. For $\lambda \geq 0$, define

$$
u_{2}(\lambda)=\frac{1}{\kappa}(\kappa / 2-2-\rho)+\frac{1}{\kappa} \sqrt{4 \kappa \lambda+(\kappa / 2-2-\rho)^{2}} .
$$

Let $\eta$ be an $\operatorname{SLE}_{\kappa}(\rho)$ with force point $v>0$. For $r>0>-r>y$, and $0<v \leq x$, define $\sigma$ to be the first time that $\eta$ hits $B(y, r)$ and $T$ to be the swallowing time of $x$. For $b \leq u_{2}(\lambda)$ and $x \geq v$, we have

$$
\mathbb{E}\left[g_{\sigma}^{\prime}(x)^{\lambda}\left(g_{\sigma}(x)-W_{\sigma}\right)^{b} \mathbb{1}_{\{\sigma<T\}}\right] \lesssim x^{u_{2}(\lambda)}(x-y-2 r)^{b-u_{2}(\lambda)},
$$

where the constant in $\lesssim$ is uniform over $x, y, r$. Assume $r<|y| \lesssim r$, define

$$
\begin{aligned}
\mathcal{F}= & \{\sigma<T, \operatorname{dist}(\eta[0, \sigma], x) \geq c x, \eta[0, \sigma] \subset B(0, C|y|), \\
& \operatorname{dist}(\eta[0, \sigma],[C y, y]) \geq c r\},
\end{aligned}
$$

where the constants $C, c$ are from Lemma 2.6. Then, for $b \leq u_{2}(\lambda)$ and $x \geq v \geq$ $(1-c) x$, we have

$$
\mathbb{E}\left[g_{\sigma}^{\prime}(x)^{\lambda}\left(g_{\sigma}(x)-W_{\sigma}\right)^{b} \mathbb{1}_{\mathcal{F}}\right] \gtrsim x^{u_{2}(\lambda)}|y|^{b-u_{2}(\lambda)},
$$

where the constant in $\gtrsim$ is uniform over $x$ and $y$.

Proof of Lemma 3.7-(3.19). We may assume $x>v$. Set

$$
M_{t}=g_{t}^{\prime}(x)^{v(v+4-\kappa) /(4 \kappa)}\left(g_{t}(x)-W_{t}\right)^{v / \kappa}\left(g_{t}(x)-g_{t}(v)\right)^{v \rho /(2 \kappa)},
$$

where $v=\kappa u_{2}(\lambda) \geq 0$. By Lemma 2.4, we know that $M$ is a local martingale for $\eta$. Note that $g_{t}(x)-g_{t}(v) \leq(x-v) g_{t}^{\prime}(x)$ and $v \rho \leq 0$. We have

$$
M_{t} \geq g_{t}^{\prime}(x)^{\lambda}\left(g_{t}(x)-W_{t}\right)^{u_{2}(\lambda)}(x-v)^{v \rho /(2 \kappa)} .
$$


Therefore,

$$
\begin{aligned}
& \mathbb{E}\left[g_{\sigma}^{\prime}(x)^{\lambda}\left(g_{\sigma}(x)-W_{\sigma}\right)^{b} \mathbb{1}_{\{\sigma<T\}}\right] \\
& \quad \leq(x-v)^{-v \rho /(2 \kappa)} M_{0} \mathbb{E}^{*}\left[\left(g_{\sigma^{*}}^{*}(x)-W_{\sigma^{*}}^{*}\right)^{b-u_{2}(\lambda)} \mathbb{1}_{\left\{\sigma^{*}<T^{*}\right\}}\right] \\
& \quad=x^{u_{2}(\lambda)} \mathbb{E}^{*}\left[\left(g_{\sigma^{*}}^{*}(x)-W_{\sigma^{*}}^{*}\right)^{b-u_{2}(\lambda)} \mathbb{1}_{\left\{\sigma^{*}<T^{*}\right\}}\right] \\
& \quad \lesssim x^{u_{2}(\lambda)}(x-y-2 r)^{b-u_{2}(\lambda)} \quad \text { by Lemma } 2.3,
\end{aligned}
$$

where $\mathbb{P}^{*}$ is the law of $\eta$ weighted by $M$ and $g^{*}, W^{*}, \sigma^{*}, T^{*}$ are defined accordingly. This implies the conclusion.

ProOf OF LEMma 3.7-(3.20). Assume the same notation as in the proof of (3.19). On $\{\operatorname{dist}(\eta[0, \sigma], x) \geq c x\}$, since $0 \leq x-v \leq c x$, by the Koebe $1 / 4$ theorem, we have $g_{t}(x)-g_{t}(v) \geq(x-v) g_{t}^{\prime}(x) / 4$. Thus

$$
M_{t} \lesssim g_{t}^{\prime}(x)^{\lambda}\left(g_{t}(x)-W_{t}\right)^{u_{2}(\lambda)}(x-v)^{v \rho /(2 \kappa)} .
$$

On $\{\eta[0, \sigma] \subset B(0, C|y|)\}$, we have $g_{\sigma}(x)-W_{\sigma} \lesssim|y|$. Therefore,

$$
\mathbb{E}\left[g_{\sigma}^{\prime}(x)^{\lambda}\left(g_{\sigma}(x)-W_{\sigma}\right)^{b_{1}} \mathbb{1}_{\mathcal{F}}\right] \gtrsim x^{u_{2}(\lambda)}|y|^{b-u_{2}(\lambda)} \mathbb{P}^{*}\left[\mathcal{F}^{*}\right],
$$

where $\mathbb{P}^{*}$ is the law of $\eta$ weighted by $M$ and $\mathcal{F}^{*}$ is defined accordingly. By Lemma 2.6, we have $\mathbb{P}^{*}\left[\mathcal{F}^{*}\right] \asymp 1$. This completes the proof.

REMARK 3.8. Taking $\lambda=b=0$ in Lemma 3.7, we see that Proposition 3.2 holds for $\mathcal{H}_{1}^{\beta}$ with $\beta_{1}^{+}=u_{2}(0)=2(\kappa / 2-2-\rho) / \kappa$.

LEMMA 3.9. Fix $\kappa \in(0,4)$ and $\rho>-2$. For $\lambda \geq 0$, define

$$
u_{3}(\lambda)=\frac{(\rho+2)}{2 \kappa}\left(\rho+4-\kappa / 2+\sqrt{4 \kappa \lambda+(\rho+4-\kappa / 2)^{2}}\right) .
$$

Let $\eta$ be an $\operatorname{SLE}_{\kappa}(\rho)$ with force point $0^{+}$. For $x>\varepsilon>0$, define $\tau$ to be the first time that $\eta$ hits $B(x, \varepsilon)$ and $T$ to be the swallowing time of $x$. Define

$$
\begin{aligned}
& \mathcal{G}=\{\tau<T, \operatorname{Im} \eta(\tau) \geq c \varepsilon\}, \\
& \mathcal{F}=\mathcal{G} \cap\{\eta[0, \tau] \subset B(0, C x), \operatorname{dist}(\eta[0, \tau],[x-\varepsilon, x+3 \varepsilon]) \geq c \varepsilon\},
\end{aligned}
$$

where $c, C$ are the constants from Lemma 2.5. Then we have

$$
\mathbb{E}\left[g_{\tau}^{\prime}(x)^{\lambda_{1}} \mathbb{1}_{\mathcal{F}}\right] \asymp \mathbb{E}\left[g_{\tau}^{\prime}(x)^{\lambda_{1}} \mathbb{1}_{\mathcal{G}}\right] \asymp x^{-u_{3}(\lambda)} \varepsilon^{u_{3}(\lambda)},
$$

where the constants in $\asymp$ are uniform over $x$ and $\varepsilon$.

Proof. Set $v=\kappa / 2-4-\rho-\sqrt{4 \kappa \lambda+(\kappa / 2-4-\rho)^{2}}$ and

$$
M_{t}=g_{t}^{\prime}(x)^{\nu(\nu+4-\kappa) /(4 \kappa)}\left(g_{t}(x)-W_{t}\right)^{\nu / \kappa}\left(g_{t}(x)-V_{t}\right)^{\nu \rho /(2 \kappa)} .
$$


Then $M$ is a local martingale and the law of $\eta$ weighted by $M$ becomes $\operatorname{SLE}_{\kappa}(\rho, v)$ with force points $\left(0^{+}, x\right)$ (see Lemma 2.4). On $\mathcal{G}$, we have $g_{\tau}(x)-W_{\tau} \asymp g_{\tau}(x)-$ $V_{\tau} \asymp \varepsilon g_{\tau}^{\prime}(x)$. Combining with the choice of $v$, we have

$$
M_{\tau} \asymp g_{\tau}^{\prime}(x)^{\lambda} \varepsilon^{-u_{3}(\lambda)} \quad \text { on } \mathcal{G} \text {. }
$$

Therefore,

$$
\mathbb{E}\left[g_{\tau}^{\prime}(x)^{\lambda_{1}} \mathbb{1}_{\mathcal{G}}\right] \asymp \varepsilon^{u_{3}(\lambda)} x^{-u_{3}(\lambda)} \mathbb{P}^{*}\left[\mathcal{G}^{*}\right], \quad \mathbb{E}\left[g_{\tau}^{\prime}(x)^{\lambda} \mathbb{1}_{\mathcal{F}}\right] \asymp \varepsilon^{u_{3}(\lambda)} x^{-u_{3}(\lambda)} \mathbb{P}^{*}\left[\mathcal{F}^{*}\right],
$$

where $\eta^{*}$ is an $\operatorname{SLE}_{\kappa}(\rho, v)$ with force points $\left(0^{+}, x\right)$, and $\mathbb{P}^{*}$ denotes its law and $\mathcal{G}^{*}, \mathcal{F}^{*}$ are defined accordingly. By Lemma 2.5 , we have $\mathbb{P}^{*}\left[\mathcal{F}^{*}\right] \asymp 1$. This completes the proof.

REMARK 3.10. Taking $\lambda=0$ in Lemma 3.9, we see that Proposition 3.3 holds for $\mathcal{H}_{1}^{\alpha}$ with $\gamma_{1}^{+}=u_{3}(0)=(\rho+2)(\rho+4-\kappa / 2) / \kappa$.

3.3. Proof of Propositions 3.1 to 3.3. Fix $\kappa \in(0,4)$ and $\rho>-2$. Suppose $\eta$ is an $\operatorname{SLE}_{\kappa}(\rho)$ with force point $v \geq 0$. We keep the same notation as before: $g_{t}$ is the conformal map from the Loewner chain, $W_{t}$ is the driving function, $V_{t}$ is the evolution of the force point and $O_{t}$ is the rightmost point of $\eta[0, t] \cap \mathbb{R}$ under $g_{t}$ and $T$ is the swallowing time of $x$. Assume $j \geq 1$.

LEMMA 3.11. If (3.3) holds for $\mathcal{H}_{2 j-1}^{\alpha}$, then (3.4) holds for $\mathcal{H}_{2 j}^{\alpha}$.

PROOF. Let $\sigma$ be the first time that $\eta$ hits the ball $B\left(y, 16(40)^{2 j-1} r\right)$. Denote $g_{\sigma}-W_{\sigma}$ by $f$. Let $\tilde{\eta}$ be the image of $\eta[\sigma, \infty)$ under $f$. We know that $\tilde{\eta}$ is an $\operatorname{SLE}_{\kappa}(\rho)$ with force point $f(v)$. Define $\tilde{\mathcal{H}}_{2 j-1}^{\alpha}$ for $\tilde{\eta}$. We have the following observations:

- Consider the image of $B(y, r)$ under $f$. By Lemma 2.2, we know that $f(B(y, r))$ is contained in the ball with center $f(y)$ and radius $4 r f^{\prime}(y)$. By the Koebe 1/4 theorem, we have

$$
|f(y)| \geq 4(40)^{2 j-1} r f^{\prime}(y) .
$$

- Consider the image of the connected component of $\partial B(x, \varepsilon) \backslash \eta[0, \sigma]$ containing $x+\varepsilon$ under $f$. By Lemma 2.1, it is contained in the ball with center $f(x+3 \varepsilon)$ and radius $8 \varepsilon f^{\prime}(x+3 \varepsilon)$. Moreover, we have

$$
f(x+3 \varepsilon)-f(v) \leq(x+3 \varepsilon-v) f^{\prime}(x+3 \varepsilon) \lesssim \varepsilon f^{\prime}(x+3 \varepsilon) .
$$

Combining these two facts with (3.3), we have

$$
\begin{aligned}
& \mathbb{P}\left[\mathcal{H}_{2 j}^{\alpha}(\varepsilon, x, y, r ; v) \mid \eta[0, \sigma]\right] \\
& \quad \leq \mathbb{P}\left[\tilde{\mathcal{H}}_{2 j-1}^{\alpha}\left(8 \varepsilon f^{\prime}(x+3 \varepsilon), f(x+3 \varepsilon), f(y), 4 r f^{\prime}(y) ; f(v)\right)\right] \\
& \quad \lesssim\left(g_{\sigma}(x+3 \varepsilon)-W_{\sigma}\right)^{\alpha_{2 j-2}^{+}-\alpha_{2 j-1}^{+}\left(\varepsilon g_{\sigma}^{\prime}(x+3 \varepsilon)\right)^{\alpha_{2 j-1}^{+}} .}
\end{aligned}
$$


By Lemma 3.7 and the fact that the swallowing time of $x+3 \varepsilon$ is greater than $T$, we have

$$
\begin{aligned}
& \mathbb{P}\left[\mathcal{H}_{2 j}^{\alpha}(\varepsilon, x, y, r ; v)\right] \\
& \lesssim \mathbb{E}\left[\left(g_{\sigma}(x+3 \varepsilon)-W_{\sigma}\right)^{\alpha_{2 j-2}^{+}-\alpha_{2 j-1}^{+}}\left(\varepsilon g_{\sigma}^{\prime}(x+3 \varepsilon)\right)^{\alpha_{2 j-1}^{+}} \mathbb{1}_{\{\sigma<T\}}\right] \\
& \lesssim \varepsilon^{\alpha_{2 j-1}^{+}}(x+3 \varepsilon)^{u_{2}\left(\alpha_{2 j-1}^{+}\right)}\left(x-y-32(40)^{2 j-1} r\right)^{\alpha_{2 j-2}^{+}-\alpha_{2 j}^{+}} \\
& \lesssim x^{\alpha_{2 j}^{+}-\alpha_{2 j-1}^{+}} \varepsilon^{\alpha_{2 j-1}^{+}} \text {. }
\end{aligned}
$$

The last line is because $x \geq \varepsilon$ and $|y| \geq(40)^{2 j} r$.

LEMMA 3.12. If (3.4) holds for $\mathcal{H}_{2 j}^{\alpha}$, then (3.3) holds for $\mathcal{H}_{2 j+1}^{\alpha}$.

PROOF. If $x \leq 64 \varepsilon$, then $\mathbb{P}\left[\mathcal{H}_{2 j+1}^{\alpha}(\varepsilon, x, y, r ; v)\right] \leq \mathbb{P}\left[\mathcal{H}_{2 j}^{\alpha}(\varepsilon, x, y, r ; v)\right]$. This gives the conclusion. In the following, we may assume $x>64 \varepsilon$. Let $\tau$ be the first time that $\eta$ hits $B(x, 16 \varepsilon)$. Denote $g_{\tau}-W_{\tau}$ by $f$. Let $\tilde{\eta}$ be the image of $\eta[\tau, \infty)$ under $f$. We know that $\tilde{\eta}$ is an $\operatorname{SLE}_{\kappa}(\rho)$ with force point $f(v)$. Define $\tilde{\mathcal{H}}_{2 j}^{\alpha}$ for $\tilde{\eta}$. We have the following observations:

- Consider the image of the connected component of $\partial B(y, r) \backslash \eta[0, \tau]$ containing $y-r$ under $f$. By Lemma 2.1, we know that it is contained in the ball with center $f(y-3 r)$ and radius $8 r f^{\prime}(y-3 r)$. By Lemma 2.3, we have

$$
|f(y-3 r)| \geq(x-y+3 r-32 \varepsilon) / 2 \geq(40)^{2 j} 8 r .
$$

- Consider the image of $B(x, \varepsilon)$ under $f$. By Lemma 2.2, we know that $B(x, \varepsilon)$ is contained in the ball with center $f(x)$ and radius $4 \varepsilon f^{\prime}(x)$. Moreover,

$$
f(x)-f(v) \leq(x-v) f^{\prime}(x) \lesssim \varepsilon f^{\prime}(x) .
$$

Combining these two facts with (3.4), we have

$$
\begin{aligned}
\mathbb{P}\left[\mathcal{H}_{2 j+1}^{\alpha}(\varepsilon, x, y, r ; v) \mid \eta[0, \tau]\right] \\
\quad \leq \mathbb{P}\left[\tilde{\mathcal{H}}_{2 j}^{\alpha}\left(4 \varepsilon f^{\prime}(x), f(x), f(y-3 r), 8 r f^{\prime}(y-3 r) ; f(v)\right)\right] \\
\quad \lesssim\left(g_{\tau}(x)-W_{\tau}\right)^{\alpha_{2 j}^{+}-\alpha_{2 j-1}^{+}\left(\varepsilon g_{\tau}^{\prime}(x)\right)^{\alpha_{2 j-1}^{+}} .}
\end{aligned}
$$

If $x=v$, by Lemma 3.4, since $\alpha_{2 j-1}^{+}$and $\alpha_{2 j}^{+}$satisfy (3.13):

we have

$$
\kappa\left(\alpha_{2 j}^{+}-\alpha_{2 j-1}^{+}\right)\left(2 \rho+4-\kappa+\kappa\left(\alpha_{2 j}^{+}-\alpha_{2 j-1}^{+}\right)\right)=4 \kappa \alpha_{2 j-1}^{+},
$$

$$
\begin{aligned}
& \mathbb{P}\left[\mathcal{H}_{2 j+1}^{\alpha}(\varepsilon, v, y, r ; v)\right] \\
& \quad \lesssim \mathbb{E}\left[\left(g_{\tau}(v)-W_{\tau}\right)^{\left.\alpha_{2 j}^{+}-\alpha_{2 j-1}^{+}\left(\varepsilon g_{\tau}^{\prime}(v)\right)^{\alpha_{2 j-1}^{+}} \mathbb{1}_{\left\{\hat{\tau}<T_{v}\right\}}\right]}\right. \\
& \quad \lesssim v^{-u_{1}\left(\alpha_{2 j}^{+}\right) \varepsilon^{\alpha_{2 j+1}^{+}}=v^{\alpha_{2 j}^{+}-\alpha_{2 j+1}^{+}} \varepsilon^{\alpha_{2 j+1}^{+}} .}
\end{aligned}
$$


For $0 \leqq x-v \lesssim \varepsilon$, we know that $B(x, \varepsilon)$ is contained in $B(v, \tilde{C} \varepsilon)$ for some constant $\overline{\tilde{C}}$, thus

$$
\begin{aligned}
& \mathbb{P}\left[\mathcal{H}_{2 j+1}^{\alpha}(\varepsilon, x, y, r ; v)\right] \\
& \quad \leq \mathbb{P}\left[\mathcal{H}_{2 j+1}^{\alpha}(\tilde{C} \varepsilon, v, y, r ; v)\right] \lesssim v^{\alpha_{2 j}^{+}-\alpha_{2 j+1}^{+}} \varepsilon^{\alpha_{2 j+1}^{+}} \lesssim x^{\alpha_{2 j}^{+}-\alpha_{2 j+1}^{+}} \varepsilon^{\alpha_{2 j+1}^{+}}
\end{aligned}
$$

This gives the conclusion.

LEMMA 3.13. If (3.5) holds for $\mathcal{H}_{2 j-1}^{\alpha}$, then (3.6) holds for $\mathcal{H}_{2 j}^{\alpha}$.

PROOF. Let $\sigma$ be the first time that $\eta$ hits $B(y, r)$. Define

$$
\begin{aligned}
\mathcal{F}= & \{\sigma<T, \operatorname{dist}(\eta[0, \sigma], x) \geq c x, \eta[0, \sigma] \subset B(0, C|y|), \\
& \operatorname{dist}(\eta[0, \sigma],[C y, y]) \geq c r\},
\end{aligned}
$$

where $c, C$ are the constants from Lemma 2.5. Denote $g_{\sigma}-W_{\sigma}$ by $f$. Let $\tilde{\eta}$ be the image of $\eta[\sigma, \infty)$ under $f$, then $\tilde{\eta}$ is an $\operatorname{SLE}_{\kappa}(\rho)$ with force point $f(v)$. Given $\eta[0, \sigma]$ and on $\mathcal{F}$, we have the following observations:

- Consider the image of $B(y, r)$ under $f$. By the Koebe $1 / 4$ theorem, it contains the ball with center $f(y)$ and radius $r f^{\prime}(y) / 4$. On $\{\operatorname{dist}(\eta[0, \sigma],[C y, y]) \geq c r\}$, we have

$$
r f^{\prime}(y) / 4 \leq|f(y)| \lesssim r f^{\prime}(y)
$$

- Consider the image of $B(x, \varepsilon)$ under $f$. On $\{\operatorname{dist}(\eta[0, \sigma], x) \geq c x\}$, by the Koebe $1 / 4$ theorem, it contains the ball with the center $f(x)$ and radius $c \varepsilon f^{\prime}(x) / 4$. Since $x-v \lesssim \varepsilon$, we have

$$
f(x)-f(v) \leq(x-v) f^{\prime}(x) \lesssim \varepsilon f^{\prime}(x) .
$$

- Compare $f(x)$ and $|f(y)| \asymp r f^{\prime}(y)$. On $\{\eta[0, \sigma] \subset B(0, C|y|)\}$, we have $f(x) \lesssim|y|$. On $\{\operatorname{dist}(\eta[0, \sigma],[C y, y]) \geq c r\}$, we have $|f(y)| \gtrsim|y|$. Thus, on $\mathcal{F}$, we have

$$
f(x) \lesssim|y| \lesssim|f(y)| \asymp r f^{\prime}(y) .
$$

Combining these three facts with (3.5), we have

$$
\begin{aligned}
\mathbb{P}\left[\mathcal{H}_{2 j}^{\alpha}(\varepsilon, x, y, r ; v) \mid \eta[0, \sigma], \mathcal{F}\right] \\
\quad \geq \mathbb{P}\left[\tilde{\mathcal{H}}_{2 j-1}^{\alpha}\left(\varepsilon f^{\prime}(x) / 4, f(x), f(y), r f^{\prime}(y) / 4 ; f(v)\right)\right] \\
\quad \gtrsim\left(g_{\sigma}(x)-W_{\sigma}\right)^{\alpha_{2 j-2}^{+}-\alpha_{2 j-1}^{+}\left(\varepsilon g_{\sigma}^{\prime}(x)\right)^{\alpha_{2 j-1}^{+}} .}
\end{aligned}
$$


By Lemma 3.7, we have

$$
\begin{aligned}
& \mathbb{P}\left[\mathcal{H}_{2 j}^{\alpha}(\varepsilon, x, y, r ; v) \cap \mathcal{F}\right] \\
& \quad \gtrsim \mathbb{E}\left[\left(g_{\sigma}(x)-W_{\sigma}\right)^{\left.\alpha_{2 j-2}^{+}-\alpha_{2 j-1}^{+}\left(\varepsilon g_{\sigma}^{\prime}(x)\right)^{\alpha_{2 j-1}^{+}} \mathbb{1}_{\mathcal{F}}\right]}\right. \\
& \quad \gtrsim x^{u_{2}\left(\alpha_{2 j-1}^{+}\right)} \varepsilon^{\alpha_{2 j-1}^{+}}=x^{\alpha_{2 j}^{+}-\alpha_{2 j-1}^{+}} \varepsilon^{\alpha_{2 j-1}^{+}} .
\end{aligned}
$$

This gives the conclusion.

LEMMA 3.14. If (3.6) holds for $\mathcal{H}_{2 j}^{\alpha}$, then (3.5) holds for $\mathcal{H}_{2 j+1}^{\alpha}$.

Proof. Let $\tau$ be the first time that $\eta$ hits $B(x, \varepsilon)$. Define $\mathcal{F}=\{\tau<T, \operatorname{Im} \eta(\tau) \geq c \varepsilon, \eta[0, \tau] \subset B(0, C x), \operatorname{dist}(\eta[0, \tau],[-C x, y+r]) \geq c r\}$, where $c, C$ are constants from Lemma 3.4. Denote $g_{\tau}-W_{\tau}$ by $f$. Let $\tilde{\eta}$ be the image of $\eta[\tau, \infty)$ under $f$, then $\tilde{\eta}$ is an $\operatorname{SLE}_{\kappa}(\rho)$ with force point $f(v)$. Define $\tilde{\mathcal{H}}_{2 j}^{\alpha}$ for $\tilde{\eta}$. Given $\eta[0, \tau]$ and on $\mathcal{F}$, we have the following observations:

- Consider the image of $B(y, r)$ under $f$. On $\mathcal{F}$, we know that $f(B(y, r))$ contains the ball with center $f(y)$ and radius $\operatorname{cr} f^{\prime}(y) / 4$; moreover, we have

$$
c r f^{\prime}(y) / 4 \leq|f(y)| \lesssim r f^{\prime}(y) .
$$

- Consider the image of $B(x, \varepsilon)$ under $f$. By the Koebe $1 / 4$ theorem, it contains the ball with center $f(x)$ and radius $\varepsilon f^{\prime}(x) / 4$. On $\{\operatorname{Im} \eta(\tau) \geq c \varepsilon\}$, we have

$$
f(x) \asymp \varepsilon f^{\prime}(x) .
$$

Since $x-v \lesssim \varepsilon$, we have

$$
f(x)-f(v) \leq(x-v) f^{\prime}(x) \lesssim \varepsilon f^{\prime}(x) .
$$

Combining these two facts with (3.6), we have

$$
\begin{aligned}
& \mathbb{P}\left[\mathcal{H}_{2 j+1}^{\alpha}(\varepsilon, x, y, r ; v) \mid \eta[0, \tau], \mathcal{F}\right] \\
& \quad \geq \mathbb{P}\left[\tilde{\mathcal{H}}_{2 j}^{\alpha}\left(\varepsilon f^{\prime}(x) / 4, f(x), f(y), r f^{\prime}(y) / 4 ; f(v)\right)\right] \gtrsim\left(\varepsilon g_{\tau}^{\prime}(x)\right)^{\alpha_{2 j}^{+}} .
\end{aligned}
$$

By Lemma 3.4, we have

$$
\begin{aligned}
& \mathbb{P}\left[\mathcal{H}_{2 j+1}^{\alpha}(\varepsilon, x, y, r ; v) \cap \mathcal{F}\right] \\
& \quad \gtrsim \mathbb{E}\left[\left(\varepsilon g_{\tau}^{\prime}(x)\right)^{\alpha_{2 j}^{+} \mathbb{1} \mathcal{F}}\right] \asymp x^{-u_{1}\left(\alpha_{2 j}^{+}\right)} \varepsilon^{u_{1}\left(\alpha_{2 j}^{+}\right)+\alpha_{2 j}^{+}}=x^{\alpha_{2 j}^{+}-\alpha_{2 j+1}^{+}} \varepsilon^{\alpha_{2 j+1}^{+}} .
\end{aligned}
$$

Proof of Proposition 3.1. Note that

$$
\alpha_{2 j+1}^{+}=\alpha_{2 j}^{+}+u_{1}\left(\alpha_{2 j}^{+}\right), \quad \alpha_{2 j}^{+}=\alpha_{2 j-1}^{+}+u_{2}\left(\alpha_{2 j-1}^{+}\right) .
$$


Combining Remark 3.6 with Lemmas 3.11, 3.12, 3.13 and 3.14, we obtain the conclusion.

ProOf OF Proposition 3.2. By Remark 3.8, we know the conclusion is true for $\mathcal{H}_{1}^{\beta}$. Note that

$$
\beta_{2 j}^{+}=\beta_{2 j-1}^{+}+u_{1}\left(\beta_{2 j-1}^{+}\right), \quad \beta_{2 j+1}^{+}=\beta_{2 j}^{+}+u_{2}\left(\beta_{2 j}^{+}\right) .
$$

Moreover, the exponents $\beta_{2 j-2}^{+}$and $\beta_{2 j-1}^{+}$satisfy (3.13):

$$
\kappa\left(\beta_{2 j-1}^{+}-\beta_{2 j-2}^{+}\right)\left(2 \rho+4-\kappa+\kappa\left(\beta_{2 j-1}^{+}-\beta_{2 j-2}^{+}\right)\right)=4 \kappa \beta_{2 j-2}^{+} .
$$

We can prove the following:

If (3.8) holds for $\mathcal{H}_{2 j-1}^{\beta}$, then (3.7) holds for $\mathcal{H}_{2 j}^{\beta}$ (by the proof of Lemma 3.12). If (3.7) holds for $\mathcal{H}_{2 j}^{\beta}$, then (3.8) holds for $\mathcal{H}_{2 j+1}^{\beta}$ (by the proof of Lemma 3.11). If (3.10) holds for $\mathcal{H}_{2 j-1}^{\beta}$, then (3.9) holds for $\mathcal{H}_{2 j}^{\beta}$ (by the proof of Lemma 3.14). If (3.9) holds for $\mathcal{H}_{2 j}^{\beta}$, then (3.10) holds for $\mathcal{H}_{2 j+1}^{\beta}$ (by the proof of Lemma 3.13). Combining all these, we complete the proof.

Proof of Proposition 3.3-(3.11)-Upper Bound. By Remark 3.10, we know that the conclusion is true for $\mathcal{H}_{1}^{\alpha}$. We will prove the conclusion for $\mathcal{H}_{2 j+1}^{\alpha}$ for $j \geq 1$. Recall that $\eta$ is an $\operatorname{SLE}_{\kappa}(\rho)$ with force point $0^{+}$. Let $\tau$ be the first time that $\eta$ hits $B(x, \varepsilon)$, and $T$ be the first time that $\eta$ swallows $x$. Recall that

$$
\mathcal{F}=\{\tau<T, \eta[0, \tau] \subset B(0, C x), \operatorname{dist}(\eta[0, \tau],[x-\varepsilon, x+3 \varepsilon]) \geq c \varepsilon\} .
$$

Given $\eta[0, \tau]$, denote $g_{\tau}-W_{\tau}$ by $f$. Let $\tilde{\eta}$ be the image of $\eta[\tau, \infty)$ under $f$, then $\tilde{\eta}$ is an $\operatorname{SLE}_{\kappa}(\rho)$ with force point $f\left(0^{+}\right)$. Define $\tilde{\mathcal{H}}_{2 j}^{\alpha}$ for $\tilde{\eta}$. We have the following observations:

- Consider the image of the connected component of $\partial B(x, \varepsilon) \backslash \eta[0, \tau]$ containing $x+\varepsilon$ under $f$. By Lemma 2.1, we know that it is contained in the ball with center $f(x+3 \varepsilon)$ and radius $8 \varepsilon f^{\prime}(x+3 \varepsilon)$. On the event $\{\operatorname{dist}(\eta[0, \tau],[x-\varepsilon, x+3 \varepsilon]) \geq$ $c \varepsilon\}$, by the Koebe distortion theorem [19], Chapter I, Theorem 1.3, we know that there exists some universal constant $\tilde{C}$ such that the ball with center $f(x+3 \varepsilon)$ and radius $8 \varepsilon f^{\prime}(x+3 \varepsilon)$ is contained in the ball with center $f(x)$ and radius $\tilde{C} \varepsilon f^{\prime}(x)$. Moreover, on the event $\{\operatorname{dist}(\eta[0, \tau],[x-\varepsilon, x+3 \varepsilon]) \geq c \varepsilon\}$, we have

$$
f(x) \asymp f(x)-f\left(0^{+}\right) \asymp \varepsilon f^{\prime}(x) .
$$

- Consider the image of the connected component of $\partial B(y, r) \backslash \eta[0, \tau]$ containing $y-r$ under $f$. By Lemma 2.1, we know that it is contained in the ball with center $f(y-3 r)$ and radius $8 r f^{\prime}(y-3 r)$. By Lemma 2.3, we know that

$$
|f(y-3 r)| \geq(x-y+3 r-2 \varepsilon) / 2 \geq|y| / 2 \geq(40)^{2 j} 8 r .
$$


Combining these two facts with (3.4), we have

$$
\mathbb{P}\left[\mathcal{H}_{2 j+1}^{\alpha}\left(\varepsilon, x, y, r ; 0^{+}\right) \mid \eta[0, \tau], \mathcal{F}\right] \lesssim\left(\varepsilon g_{\tau}^{\prime}(x)\right)^{\alpha_{2 j}^{+}}
$$

By Lemma 3.9, we have

$$
\mathbb{P}\left[\mathcal{H}_{2 j+1}^{\alpha}\left(\varepsilon, x, y, r ; 0^{+}\right) \cap \mathcal{F}\right] \lesssim \mathbb{E}\left[\left(\varepsilon g_{\tau}^{\prime}(x)\right)^{\alpha_{2 j}^{+}} \mathbb{1}_{\mathcal{F}}\right] \asymp \varepsilon^{u_{3}\left(\alpha_{2 j}^{+}\right)+\alpha_{2 j}^{+}} .
$$

Note that

$$
\gamma_{2 j+1}^{+}=u_{3}\left(\alpha_{2 j}^{+}\right)+\alpha_{2 j}^{+}
$$

This completes the proof.

Proof of Proposition 3.3-(3.11)-Lower Bound. Assume the same notation as in the proof of the upper bound. We have the following observations:

- Consider the image of $B(x, \varepsilon)$ under $f$. By the Koebe $1 / 4$ theorem, it contains the ball with center $f(x)$ and radius $\varepsilon f^{\prime}(x) / 4$. Moreover, on the event $\mathcal{F}$, we have

$$
f(x) \asymp f(x)-f\left(0^{+}\right) \asymp \varepsilon f^{\prime}(x) .
$$

- Consider the image of $B(y, r)$ under $f$. Note that $r \geq C x$ and $|y| \geq(40)^{2 j+1} r$. Thus, on the event $\{\eta[0, \tau] \subset B(0, C x)\}$, we know that $\eta[0, \tau]$ does not hit $B(y, r)$. Thus $f(B(y, r))$ contains the ball with center $f(y)$ and radius $r f^{\prime}(y) / 4$. On the event $\{\eta[0, \tau] \subset B(0, C x)\}$, we know that

$$
r f^{\prime}(y) / 4 \leq|f(y)| \leq|y|+(C x)^{2} /|y| \leq 2|y| \asymp r .
$$

Combining these two facts with (3.6), we have

$$
\mathbb{P}\left[\mathcal{H}_{2 j+1}^{\alpha}\left(\varepsilon, x, y, r ; 0^{+}\right) \mid \eta[0, \tau], \mathcal{F}\right] \gtrsim\left(\varepsilon g_{\tau}^{\prime}(x)\right)^{\alpha_{2 j}^{+}} .
$$

By Lemma 3.9, we have

$$
\mathbb{P}\left[\mathcal{H}_{2 j+1}^{\alpha}\left(\varepsilon, x, y, r ; 0^{+}\right) \cap \mathcal{F}\right] \gtrsim \mathbb{E}\left[\left(\varepsilon g_{\tau}^{\prime}(x)\right)^{\alpha_{2 j}^{+}} \mathbb{1}_{\mathcal{F}}\right] \asymp \varepsilon^{u_{3}\left(\alpha_{2 j}^{+}\right)+\alpha_{2 j}^{+}} .
$$

This completes the proof.

Proof of Proposition 3.3-(3.12). By the same proof of (3.11), we can prove that

$$
\mathbb{P}\left[\mathcal{H}_{2 j}^{\alpha}\left(\varepsilon, x, y, r ; 0^{+}\right) \cap \mathcal{F}\right] \asymp \mathbb{E}\left[\left(\varepsilon g_{\tau}^{\prime}(x)\right)^{\beta_{2 j-1}^{+}}\right] \asymp \varepsilon^{u_{3}\left(\beta_{2 j-1}^{+}\right)+\beta_{2 j-1}^{+}} .
$$

Note that

$$
\gamma_{2 j}^{+}=u_{3}\left(\beta_{2 j-1}^{+}\right)+\beta_{2 j-1}^{+} .
$$

This completes the proof. 
4. SLE interior arm exponents. Fix $\kappa \in(0,4)$ and let $\eta$ be an $\operatorname{SLE}_{\kappa}$ in $\mathbb{H}$ from 0 to $\infty$. We keep the same notation as before: $g_{t}$ is the family of conformal maps for the Loewner chain, $W_{t}$ is the driving function. We write c.c. for "connected component."

Fix $z \in \mathbb{H}$ with $|z|=1$ and suppose $r>0$ and $y \leq-4 r$. Let $\tau_{1}$ be the first time that $\eta$ hits $B(z, \varepsilon)$. Define

$$
\mathcal{E}_{2}(\varepsilon, z)=\left\{\tau_{1}<\infty\right\} .
$$

Let $\sigma_{1}$ be the first time after $\tau_{1}$ that $\eta$ hits the c.c. of $\partial B(y, r) \backslash \eta\left[0, \tau_{1}\right]$ containing $y-r$. Define $\mathcal{E}^{g}$ to be the event that $z$ is in the unbounded c.c. of $\mathbb{H} \backslash\left(\eta\left[0, \sigma_{1}\right] \cup\right.$ $B(y, r))$.

Given $\eta\left[0, \sigma_{1}\right]$, we know that $B(z, \varepsilon) \backslash \eta\left[0, \sigma_{1}\right]$ has one c.c. that contains $z$, denoted by $C_{z}$. The boundary $\partial C_{z}$ consists of pieces of $\eta\left[0, \sigma_{1}\right]$ and pieces of $\partial B(z, \varepsilon)$. Consider $\partial C_{z} \cap \partial B(z, \varepsilon)$. There may be several c.c.s, but there is only one which can be connected to $\infty$ in $\mathbb{H} \backslash\left(\eta\left[0, \sigma_{1}\right] \cup B(z, \varepsilon)\right)$. We denote this c.c. by $C_{z}^{b}$, oriented it clockwise and denote the end point as $X_{z}^{b}$; see Figure 2 .

Let $\tau_{2}$ be the first time after $\sigma_{1}$ that $\eta$ hits $C_{z}^{b}$, and let $\sigma_{2}$ be the first time after $\tau_{2}$ that $\eta$ hits the c.c. of $\partial B(y, r) \backslash \eta\left[0, \tau_{2}\right]$ containing $y-r$. For $j \geq 2$, let $\tau_{j}$ be the first time after $\sigma_{j-1}$ such that $\eta$ hits the c.c. of $C_{z}^{b} \backslash \eta\left[0, \sigma_{j-1}\right]$ containing $X_{z}^{b}$ and let $\sigma_{j}$ be the first time after $\tau_{j}$ that $\eta$ hits the c.c. of $\partial B(y, r) \backslash \eta\left[0, \tau_{j}\right]$ containing $y-r$. For $j \geq 2$, define

$$
\mathcal{E}_{2 j}(\varepsilon, z, y, r)=\mathcal{E}^{g} \cap\left\{\tau_{j}<T_{z}\right\} .
$$

We will prove the following estimate on the probability of $\mathcal{E}_{2 j}$.

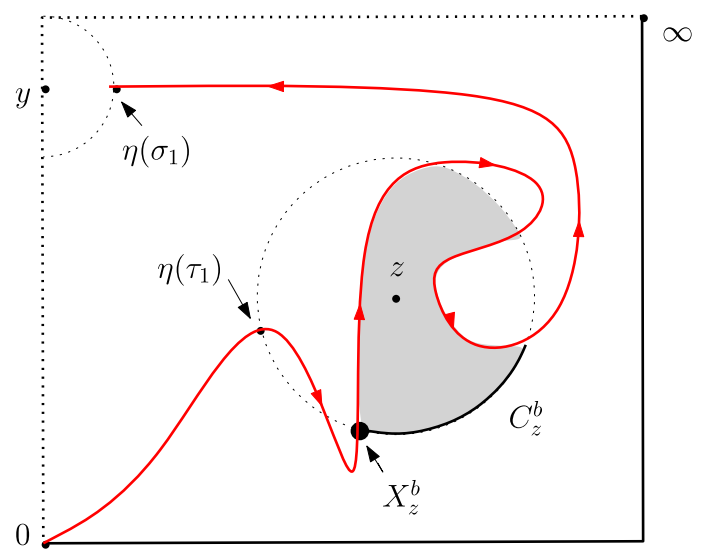

FIG. 2. The gray part is the c.c. of $B(z, \varepsilon) \backslash \eta\left[0, \sigma_{1}\right]$ that contains $z$, which is denoted by $C_{z}$. The bold part of $\partial C_{z}$ is $C_{z}^{b}$. The point $X_{z}^{b}$ is denoted in the figure. 
Proposition 4.1. Fix $\kappa \in(0,4)$ and $z \in \mathbb{H}$ with $|z|=1$. For $j \geq 1$, define

$$
\alpha_{2 j}=\left(16 j^{2}-(\kappa-4)^{2}\right) /(8 \kappa) .
$$

Define $\mathcal{F}=\left\{\eta\left[0, \tau_{1}\right] \subset B(0, R)\right\}$ where $R$ is the constant from Lemma 4.2. Then we have, for $j \geq 1$,

$$
\begin{aligned}
\mathbb{P}\left[\mathcal{E}_{2 j}(\varepsilon, z, y, r) \cap \mathcal{F}\right]= & \varepsilon^{\alpha_{2 j}+o(1)}, \\
& \text { provided } R \leq r \leq(40)^{2 j} r \leq|y| \lesssim r .
\end{aligned}
$$

We will first explain the choice of the constant $R$ in Lemma 4.2, and then prove the lower bound and the upper bound of (4.1) separately. The lower bound is easier, and the upper bound requires the estimates in Lemmas 4.3 and 4.4 .

Lemma 4.2. Fix $\kappa \in(0,8)$ and let $\eta$ be an $\operatorname{SLE}_{\kappa}$ in $\mathbb{H}$ from 0 to $\infty$. For $\lambda \geq 0$, define

$$
\begin{gathered}
\rho=\kappa / 2-4-\sqrt{4 \kappa \lambda+(\kappa / 2-4)^{2}}, \\
v(\lambda)=\frac{1}{2}-\frac{\kappa}{16}-\frac{\lambda}{2}+\frac{1}{8} \sqrt{4 \kappa \lambda+(\kappa / 2-4)^{2}} .
\end{gathered}
$$

Fix $z \in \mathbb{H}$ with $|z|=1$. For $\varepsilon>0$, let $\tau$ be the first time that $\eta$ hits $B(z, \varepsilon)$. Define $\Theta_{t}=\arg \left(g_{t}(z)-W_{t}\right)$. For $\delta \in(0,1 / 16), R \geq 4$, define

$$
\mathcal{G}=\left\{\tau<\infty, \Theta_{\tau} \in(\delta, \pi-\delta)\right\}, \quad \mathcal{F}=\{\eta[0, \tau] \subset B(0, R)\} .
$$

There exists a constant $R$ depending only on $\kappa$ and $z$ such that the following is true:

$$
\varepsilon^{v(\lambda)} \lesssim \mathbb{E}\left[\left|g_{\tau}^{\prime}(z)\right|^{\lambda} \mathbb{1}_{\mathcal{F} \cap \mathcal{G}}\right] \leq \mathbb{E}\left[\left|g_{\tau}^{\prime}(z)\right|^{\lambda_{1}} \mathbb{1}_{\mathcal{G}}\right] \lesssim \varepsilon^{v(\lambda)} \delta^{-v(\lambda)-\rho^{2} /(2 \kappa)},
$$

where the constants in $\lesssim$ are uniform over $\varepsilon, \delta$.

PRoof. Similar results were proved in [23], Section 6.3, and [18], Lemmas $4.1,4.2$, with constants in $\lesssim$ only uniform over $\varepsilon$. In our setting, we need the explicit dependence on $\delta$. Set

$$
M_{t}=\left|g_{t}^{\prime}(z)\right|^{\rho(\rho+8-2 \kappa) /(8 \kappa)}\left(\operatorname{Im} g_{t}(z)\right)^{\rho^{2} /(8 \kappa)}\left|g_{t}(z)-W_{t}\right|^{\rho / \kappa} .
$$

From [21], Theorem 6, the process $M$ is a local martingale and the law of $\eta$ weighted by $M$ becomes the law of $\operatorname{SLE}_{\kappa}(\rho)$ with force point $z$. We introduce two other quantities:

$$
\Upsilon_{t}=\frac{\operatorname{Im} g_{t}(z)}{\left|g_{t}^{\prime}(z)\right|}, \quad S_{t}=\sin \Theta_{t}=\frac{\operatorname{Im} g_{t}(z)}{\left|g_{t}(z)-W_{t}\right|} .
$$


Then we can rewrite $M$ as follows:

$$
M_{t}=\left|g_{t}^{\prime}(z)\right|^{\lambda} \Upsilon_{t}^{-v(\lambda)} S_{t}^{v(\lambda)+\rho^{2} /(8 \kappa)} .
$$

By the Koebe $1 / 4$ theorem, we know that $\Upsilon_{\tau} \asymp \varepsilon$. On $\mathcal{G}$, we know that $S_{\tau} \geq \delta / 2$ for $\delta<1 / 16$. Thus

$$
\varepsilon^{v(\lambda)} \mathbb{P}^{*}\left[\mathcal{F}^{*} \cap \mathcal{G}^{*}\right] \lesssim \mathbb{E}\left[\left|g_{t}^{\prime}(z)\right|^{\lambda} \mathbb{1}_{\mathcal{F} \cap \mathcal{G}}\right] \leq \mathbb{E}\left[\left|g_{t}^{\prime}(z)\right|^{\lambda} \mathbb{1}_{\mathcal{G}}\right] \lesssim \varepsilon^{v(\lambda)} \delta^{-v(\lambda)-\rho^{2} /(8 \kappa)},
$$

where $\eta^{*}$ is an $\operatorname{SLE}_{\kappa}(\rho)$ with force point $z, \mathbb{P}^{*}$ denotes its law and $\tau^{*}, \Theta^{*}, \mathcal{F}^{*}, \mathcal{G}^{*}$ are defined accordingly. By [18], equation (4.7), (4.8), we have

$$
\begin{aligned}
& \mathbb{P}^{*}\left[\eta^{*}\left[0, \tau^{*}\right] \subset B(0, R)\right] \rightarrow 1, \quad \text { as } R \rightarrow \infty \quad \text { and } \\
& \mathbb{P}^{*}\left[\Theta_{\tau^{*}}^{*} \in(1 / 16, \pi-1 / 16)\right] \asymp 1 .
\end{aligned}
$$

Therefore, there exists a constant $R$ depending only on $\kappa$ and $z$ such that

$$
\mathbb{P}^{*}\left[\mathcal{F}^{*} \cap \mathcal{G}^{*}\right] \geq \mathbb{P}^{*}\left[\eta^{*}\left[0, \tau^{*}\right] \subset B(0, R), \Theta_{\tau^{*}}^{*} \in(1 / 16, \pi-1 / 16)\right] \asymp 1 .
$$

This completes the proof.

We will fix the constant $R$ from Lemma 4.2 in the following of the section. The conclusion for $\mathcal{E}_{2}$ was proved in [4], Proposition 4, we will prove the conclusion for $\mathcal{E}_{2 j+2}$ for $j \geq 1$. We will need the following conclusion from Section 3 . For $j \geq 1$, taking $\rho=0$ in Proposition 3.1, we have $\alpha_{2 j}^{+}=2 j(2 j+4-\kappa / 2) / \kappa$ and

$$
\mathbb{P}\left[\mathcal{H}_{2 j}^{\alpha}(\varepsilon, x, y, r)\right] \asymp x^{\alpha_{2 j}^{+}-\alpha_{2 j-1}^{+}} \varepsilon^{\alpha_{2 j-1}^{+}} \quad \text { provided }(40)^{2 j} r \leq|y| \lesssim r .
$$

Note that, since $\rho=0$, we may assume $v=x$ and we eliminate the force point in the definition of $\mathcal{H}_{2 j}^{\alpha}$.

Proof of Proposition 4.1-(4.1)-Lower Bound. We will prove the lower bound for the probability of $\mathcal{E}_{2 j+2}$. Let $\eta$ be an $\mathrm{SLE}_{\kappa}$ in $\mathbb{H}$ from 0 to $\infty$. Let $\tau$ be the first time that $\eta$ hits $B(z, \varepsilon)$. Denote the centered conformal map $g_{t}-W_{t}$ by $f_{t}$ for $t \geq 0$. Recall that $\mathcal{F}=\{\eta[0, \tau] \subset B(0, R)\}$. Fix some $\delta>0$ and define $\mathcal{G}=\mathcal{F} \cap\left\{\Theta_{\tau} \in(\delta, \pi-\delta)\right\}$.

We run $\eta$ until the time $\tau$. On $\mathcal{G}$, by the Koebe $1 / 4$ theorem, we know that $f_{\tau}(B(z, \varepsilon))$ contains the ball with center $w:=f_{\tau}(z)$ and radius $u:=\varepsilon\left|f_{\tau}^{\prime}(z)\right| / 4$ and

$$
\arg (w) \in(\delta, \pi-\delta), \quad u \leq \operatorname{Im} w \leq 16 u .
$$

We wish to apply (4.2), however, this ball is centered at $w=f_{\tau}(z)$ which does not satisfy the conditions in (4.2). We will fix this problem by running $\eta$ a little further and argue that there is a positive chance that $\eta$ does the right thing.

Let $\tilde{\eta}$ be the image of $\eta[\tau, \infty)$ under $f_{\tau}$. Let $\gamma$ be the broken line from 0 to $w$ and then to $-u+u i$ and let $A_{u}$ be the $u / 4$-neighborhood of $\gamma$. Let $S_{1}$ be the first 
time that $\tilde{\eta}$ exits $A_{u}$ and let $S_{2}$ be the first time that $\tilde{\eta}$ hits the ball with center $-u+$ $u i$ and radius $u / 4$. By [18], Lemma 2.5 , we know that $\mathbb{P}\left[S_{2}<S_{1}\right]$ is bounded from below by positive constant depending only on $\kappa$ and $\delta$. On $\left\{S_{2}<S_{1}\right\}$, it is clear that there exist constants $x_{\delta}, c_{\delta}>0$ depending only on $\delta$ such that $f_{S_{2}}(B(z, \varepsilon))$ contains the ball with center $x_{\delta} u$ and radius $c_{\delta} u$.

Consider the image of $B(y, r)$ under $f_{S_{2}}$. On $\mathcal{F} \cap\left\{S_{2}<S_{1}\right\}$, we know that the image of $B(y, r)$ under $f_{S_{2}}$ contains the ball with center $f_{S_{2}}(y)$ and radius $r f_{S_{2}}^{\prime}(y) / 4$ where

$$
2 y \leq f_{S_{2}}(y) \leq y, \quad f_{S_{2}}^{\prime}(y) \asymp 1 .
$$

Combining with (4.2), we have

$$
\mathbb{P}\left[\mathcal{E}_{2 j+2}(\varepsilon, z, y, r) \mid \eta\left[0, S_{2}\right], \mathcal{G} \cap\left\{S_{2}<S_{1}\right\}\right] \gtrsim\left(\varepsilon\left|g_{\tau}^{\prime}(z)\right|\right)^{\alpha_{2 j}^{+}} .
$$

Since $\left\{S_{2}<S_{1}\right\}$ has a positive chance, we have

$$
\mathbb{P}\left[\mathcal{E}_{2 j+2}(\varepsilon, z, y, r) \mid \eta[0, \tau], \mathcal{G}\right] \gtrsim\left(\varepsilon\left|g_{\tau}^{\prime}(z)\right|\right)^{\alpha_{2 j}^{+}} .
$$

Therefore, by Lemma 4.2, we have

$$
\mathbb{P}\left[\mathcal{E}_{2 j+2}(\varepsilon, z, y, r)\right] \gtrsim \mathbb{E}\left[\left(\varepsilon\left|g_{\tau}^{\prime}(z)\right|\right)^{\alpha_{2 j}^{+}} \mathbb{1}_{\mathcal{G}}\right] \asymp \varepsilon^{v\left(\alpha_{2 j}^{+}\right)+\alpha_{2 j}^{+}}=\varepsilon^{\alpha_{2 j+2}},
$$

where the constants in $\gtrsim$ and $\asymp$ are uniform over $\varepsilon$. This completes the proof.

LeMma 4.3. Fix $\kappa \in(0,4)$ and let $\eta$ be an $\mathrm{SLE}_{\kappa}$ in $\mathbb{H}$ from 0 to $\infty$. Fix $z \in \mathbb{H}$ with $|z|=1$. Let $\Theta_{t}=\arg \left(g_{t}(z)-W_{t}\right)$. For $C \geq 16$, let $\xi$ be the first time that $\eta$ hits $\partial B(z, C \varepsilon)$. For $\delta \in(0,1 / 16)$, define

$$
\mathcal{F}=\left\{\xi<\infty, \Theta_{\xi} \in(\delta, \pi-\delta), \eta[0, \xi] \subset B(0, R)\right\} .
$$

Then we have

$$
\mathbb{P}\left[\mathcal{E}_{2 j+2}(\varepsilon, z, y, r) \cap \mathcal{F}\right] \lesssim C^{A} \delta^{-B} \varepsilon^{\alpha_{2 j+2}} \quad \text { provided } y \leq-20 r, r \geq R,
$$

where $A, B$ are some constants depending on $\kappa$ and $j$, and the constant in $\lesssim i s$ uniform over $\delta, C, \varepsilon$.

PRoof. We run the curve up to time $\xi$ and let $f=g_{\xi}-W_{\xi}$. We have the following observations:

- Consider $f(B(z, \varepsilon))$. By Lemma 2.2, we know that $f(B(z, \varepsilon))$ is contained in the ball with center $f(z)$ and radius $u:=4 \varepsilon\left|f^{\prime}(z)\right|$. Applying the Koebe $1 / 4$ theorem to $f$, we have

$$
C \varepsilon\left|f^{\prime}(z)\right| / 4 \leq \operatorname{Im} f(z) \leq 4 C \varepsilon\left|f^{\prime}(z)\right| .
$$

Next, we argue that $f(B(z, \varepsilon))$ is contained in the ball with center $|f(z)| \in \mathbb{R}$ and radius $8 C u / \delta$. Since $f((z, \varepsilon))$ is contained in the ball with center $f(z)$ and 
radius $u$, it is clear that $f(B(z, \varepsilon))$ is contained in the ball with center $|f(z)|$ with radius $u+2|f(z)|$. By (4.3), we have

$$
C u / 16 \leq|f(z)| \sin \Theta_{\xi} \leq C u .
$$

Since $\Theta_{\xi} \in(\delta, \pi-\delta)$, we know that, for $\delta>0$ small, we have $\sin \Theta_{\xi} \geq \delta / 2$. Thus, $C u / 16 \leq|f(z)| \leq 2 C u / \delta$. Therefore, $f(B(z, \varepsilon))$ is contained in the ball with center $|f(z)|$ with radius $8 C u / \delta$. In summary, we know that $f(B(z, \varepsilon))$ is contained in the ball with center $|f(z)|$ and radius $32 C \varepsilon\left|f^{\prime}(z)\right| / \delta$ where

$$
C \varepsilon\left|f^{\prime}(z)\right| / 4 \leq|f(z)| \leq 8 C \varepsilon\left|f^{\prime}(z)\right| / \delta \text {. }
$$

- Consider $f(B(y, r))$. Since $\{\eta[0, \xi] \subset B(0, R)\}$ and $y \leq-20 r$ with $r \geq R$, we know that $f(B(y, r))$ is contained in the ball with center $f(y)$ and radius $4 r f^{\prime}(y)$ where

$$
2 y \leq f(y) \leq y, \quad f^{\prime}(y) \asymp 1 .
$$

Combining these two facts with (4.2), we have

$$
\mathbb{P}\left[\mathcal{E}_{2 j+2}(\varepsilon, z, y, r) \mid \eta[0, \xi], \mathcal{F}\right] \lesssim\left(C \varepsilon\left|f^{\prime}(z)\right| / \delta\right)^{\alpha_{2 j}^{+}},
$$

where the constant in $\lesssim$ is uniform over $C, \varepsilon, \delta$. Thus, by Lemma 4.2, we have

$$
\mathbb{P}\left[\mathcal{E}_{2 j+2}(\varepsilon, z, y, r) \cap \mathcal{F}\right] \lesssim C^{A} \delta^{-B} \varepsilon^{\alpha_{2 j+2}},
$$

where $A, B$ are some constants depending on $\kappa$ and $j$. This completes the proof.

LEMMA 4.4. Fix $\kappa \in(0,8)$ and let $\eta$ be an $\mathrm{SLE}_{\kappa}$ in $\mathbb{H}$ from 0 to $\infty$. Fix $z \in \mathbb{H}$ with $|z|=1$. Let $T_{z}$ be the first time that $\eta$ swallows $z$ and set $\Theta_{t}=\arg \left(g_{t}(z)-W_{t}\right)$. Take $n \in \mathbb{N}$ such that $B\left(z, 16 \varepsilon 2^{n}\right)$ is contained in $\mathbb{H}$. For $1 \leq m \leq n$, let $\xi_{m}$ be the first time that $\eta$ hits $B\left(z, 16 \varepsilon 2^{n-m+1}\right)$. Note that $\xi_{1}, \ldots, \xi_{n}$ is an increasing sequence of stopping times and $\xi_{1}$ is the first time that $\eta$ hits $B\left(z, 16 \varepsilon 2^{n}\right)$ and $\xi_{n}$ is the first time that $\eta$ hits $B(z, 32 \varepsilon)$. For $1 \leq m \leq n$, for $\delta>0$, define

$$
\mathcal{F}_{m}=\left\{\xi_{m}<T_{z}, \Theta_{\xi_{m}} \notin(\delta, \pi-\delta)\right\}
$$

There exists a function $p:(0,1) \rightarrow[0,1]$ with $p(\delta) \downarrow 0$ as $\delta \downarrow 0$ such that

$$
\mathbb{P}\left[\bigcap_{1}^{n} \mathcal{F}_{m}\right] \leq p(\delta)^{n} \text {. }
$$

PROOF. For $w \in \mathbb{H}$ with $\arg (w) \notin(\delta, \pi-\delta)$, by (A.3), we know that

$$
\mathbb{P}[\eta \text { hits } B(w, \operatorname{Im} w)] \leq C \delta^{8 / \kappa-1},
$$

where $C$ is some universal constant.

For $1 \leq m \leq n$, let $f_{m}=g_{\xi_{m}}-W_{\xi_{m}}$. Note that $\xi_{m}$ is the first time that $\eta$ hits $B\left(z, 16 \varepsilon 2^{n-m+1}\right)$. We denote $\varepsilon 2^{n-m+1}$ by $u$. By Lemma 2.2, we know that the 
ball $f_{m}(B(z, u))$ is contained in the ball with center $f_{m}(z)$ and radius $4 u\left|f_{m}^{\prime}(z)\right|$; moreover,

$$
4 u\left|f_{m}^{\prime}(z)\right| \leq \operatorname{Im} f_{m}(z) \leq 64 u\left|f_{m}^{\prime}(z)\right| .
$$

Therefore, by (4.4), we have $\mathbb{P}\left[\mathcal{F}_{m+4} \mid \eta\left[0, \xi_{m}\right]\right] \leq C \delta^{8 / \kappa-1}$. Iterating this inequality, we have

$$
\mathbb{P}\left[\bigcap_{1}^{n} \mathcal{F}_{m}\right] \leq\left(C \delta^{8 / \kappa-1}\right)^{n / 4},
$$

where $C$ is some universal constant. This implies the conclusion.

Proof of Proposition 4.1-(4.1)-UPPer Bound. Assume the same notation as in Lemma 4.4. Recall that $\mathcal{F}=\left\{\eta\left[0, \tau_{1}\right] \subset B(0, R)\right\}$. By Lemma 4.3, we have, for $1 \leq m \leq n$

$$
\mathbb{P}\left[\mathcal{E}_{2 j+2} \cap \mathcal{F} \cap \mathcal{F}_{m}^{c}\right] \lesssim 2^{n A} \delta^{-B} \varepsilon^{\alpha_{2 j+2}},
$$

where $A, B$ are some constants depending on $\kappa$ and $j$. Combining with Lemma 4.4, we have, for any $n$ and $\delta>0$,

$$
\mathbb{P}\left[\mathcal{E}_{2 j+2}(\varepsilon, z, y, r) \cap \mathcal{F}\right] \lesssim n 2^{n A} \delta^{-B} \varepsilon^{\alpha_{2 j+2}}+p(\delta)^{n},
$$

where $p(\delta) \downarrow 0$ as $\delta \downarrow 0$. This implies the conclusion.

\section{Ising model.}

5.1. Definitions. We focus on the square lattice $\mathbb{Z}^{2}$. Two vertices $x=\left(x_{1}, x_{2}\right)$ and $y=\left(y_{1}, y_{2}\right)$ are neighbors if $\left|x_{1}-y_{1}\right|+\left|x_{2}-y_{2}\right|=1$, and we write $x \sim y$. We denote by $\Lambda_{n}(x)$ the box centered at $x$ :

$$
\Lambda_{n}(x)=x+[-n, n]^{2}, \quad \Lambda_{n}=\Lambda_{n}(0) .
$$

Let $\Omega$ be a finite subset of $\mathbb{Z}^{2}$, and the edge-set of $\Omega$ consists of all edges of $\mathbb{Z}^{2}$ that link two vertices of $\Omega$. The boundary of $\Omega$ is defined to be $\partial \Omega=\{e=$ $(x, y): x \sim y, x \in \Omega, y \notin \Omega\}$. We sometimes identify a boundary edge $(x, y)$ with one of its endpoints. Two vertices $x=\left(x_{1}, x_{2}\right)$ and $y=\left(y_{1}, y_{2}\right)$ are $\star$-neighbors if $\max \left\{\left|x_{1}-y_{1}\right|,\left|x_{2}-y_{2}\right|\right\}=1$. With this definition, each vertex has eight $\star-$ neighbors instead of four.

The Ising model with free boundary conditions is a random assignment $\sigma \in$ $\{\ominus, \oplus\}^{\Omega}$ of spins $\sigma_{x} \in\{\ominus, \oplus\}$, where $\sigma_{x}$ denotes the spin at the vertex $x$. The Hamiltonian of the Ising model is defined by $H_{\Omega}^{\text {free }}(\sigma)=-\sum_{x \sim y} \sigma_{x} \sigma_{y}$. The Ising measure is the Boltzmann measure with Hamiltonian $H_{\Omega}^{\text {free }}$ and inversetemperature $\beta>0$ :

$$
\mu_{\beta, \Omega}^{\text {free }}[\sigma]=\frac{\exp \left(-\beta H_{\Omega}^{\text {free }}(\sigma)\right)}{Z_{\beta, \Omega}^{\text {free }}} \quad \text { where } Z_{\beta, \Omega}^{\text {free }}=\sum_{\sigma} \exp \left(-\beta H_{\Omega}^{\text {free }}(\sigma)\right) .
$$


For a graph $\Omega$ and $\tau \in\{\ominus, \oplus\}^{\mathbb{Z}^{2}}$, one may also define the Ising model with boundary conditions $\tau$ by the Hamiltonian

$$
H_{\Omega}^{\tau}(\sigma)=-\sum_{x \sim y,\{x, y\} \cap \Omega \neq \varnothing} \sigma_{x} \sigma_{y} \quad \text { if } \sigma_{x}=\tau_{x}, \forall x \notin \Omega .
$$

The Ising model has the following domain Markov property: Suppose $\Omega \subset \Omega^{\prime}$ are two finite subsets of $\mathbb{Z}^{2}$. Let $\tau \in\{\ominus, \oplus\}^{\mathbb{Z}^{2}}$ and let $\beta>0$. Suppose $X$ is a random variable measurable with respect to $\left\{\sigma_{x}: x \in \Omega\right\}$. Then we have

$$
\mu_{\beta, \Omega^{\prime}}^{\tau}\left[X \mid \sigma_{x}=\tau_{x}, \forall x \in \Omega^{\prime} \backslash \Omega\right]=\mu_{\beta, \Omega}^{\tau}[X] .
$$

Dobrushin domains are the discrete analogue of simply connected domains with two marked points on their boundary. Suppose $(\Omega ; a, b)$ is a Dobrushin domain. Assume that $\partial \Omega$ can be divided into two $\star$-connected paths from $a$ to $b$ (counterclockwise) and from $b$ to $a$. Several boundary conditions will be of particular interest in this paper:

- We denote by $\mu^{\text {free }}$ for free boundary conditions. We denote by $\mu^{\oplus}$ (resp. $\mu^{\ominus}$ ) for the boundary conditions $\tau_{x}=\oplus$ for all $x$ (resp., $\tau_{x}=\ominus$ for all $x$ ).

- $(\ominus \oplus)$ boundary conditions: $\oplus$ along $\partial \Omega$ from $a$ to $b$, and $\ominus$ along $\partial \Omega$ from $b$ to $a$. These boundary conditions are also called Dobrushin boundary conditions, or domain-wall boundary conditions.

- ( $\ominus$ free) boundary conditions: free along $\partial \Omega$ from $a$ to $b$, and $\ominus$ along $\partial \Omega$ from $b$ to $a$.

The set $\{\ominus, \oplus\}^{\Omega}$ is equipped with a partial order: $\sigma \leq \sigma^{\prime}$ if $\sigma_{x} \leq \sigma_{x}^{\prime}$ for all $x \in \Omega$. A random variable $X$ is increasing if $\sigma \leq \sigma^{\prime}$ implies $X(\sigma) \leq X\left(\sigma^{\prime}\right)$. An event $\mathcal{A}$ is increasing if $\mathbb{1}_{\mathcal{A}}$ is increasing. The following inequality is the FKG inequality for the Ising model: Let $\Omega$ be a finite subset, let $\tau$ be the boundary conditions, and let $\beta>0$. For any two increasing events $\mathcal{A}$ and $\mathcal{B}$, we have

$$
\mu_{\beta, \Omega}^{\tau}[\mathcal{A} \cap \mathcal{B}] \geq \mu_{\beta, \Omega}^{\tau}[\mathcal{A}] \mu_{\beta, \Omega}^{\tau}[\mathcal{B}]
$$

As a consequence of the FKG inequality, we have the following comparison between boundary conditions: For boundary conditions $\tau_{1} \leq \tau_{2}$ and an increasing event $\mathcal{A}$, we have

$$
\mu_{\beta, \Omega}^{\tau_{1}}[\mathcal{A}] \leq \mu_{\beta, \Omega}^{\tau_{2}}[\mathcal{A}] .
$$

The Ising model with inverse-temperature $\beta>0$ is related to the random-cluster model with parameters $(p, 2)$ through the Edwards-Sokal coupling, thus the critical value $p_{c}(2)$ for the random-cluster model gives the critical value of $\beta$ : $\beta_{c}=(1 / 2) \log (1+\sqrt{2})$. We focus on the critical Ising model on the square lattice and derive the arm exponents. To this end, we need three inputs:

- The convergence of the scaling limit of the interface in the critical Ising model. This is proved in $[8,12]$; see Theorems 5.7 and 5.8. 
- The arm exponents of $\mathrm{SLE}_{3}$. This is the topic of Sections 3 and 4.

- A stronger version of Russo-Seymour-Welsh inequality for the critical Ising model. This is proved in [7]; see Proposition 5.1. We can deduce the so-called quasi-multiplicativity from the RSW inequality.

With these three inputs at hand, we can apply the same strategy as in [22] where the authors derived the arm exponents of the critical percolation. The content in this section is not new, and we just summarize the known results and explain how to put them together to get the arm exponents of the critical Ising model.

5.2. Quasi-multiplicativity. In this section, we first introduce a stronger version of RSW inequality-Proposition 5.1-for the critical Ising model and then define quasi-multiplicativity for the model. The quasi-multiplicativity is a consequence of the RSW inequality and, roughly speaking, it guarantees that we can use the crossing events of $\mathrm{SLE}_{3}$ to approximate the crossing events of the Ising model.

A discrete topological rectangle $(\Omega ; a, b, c, d)$ is a bounded simply-connected subdomain of $\mathbb{Z}^{2}$ with four marked boundary points. The four points are in counterclockwise order and $(a b)$ denotes the arc of $\partial \Omega$ from $a$ to $b$. We denote by $d_{\Omega}((a b),(c d))$ the discrete extermal distance between $(a b)$ and $(c d)$ in $\Omega$; see [6], Section 6. The discrete extremal distance is uniformly comparable to and converges to its continuous counterpart-the classical extremal distance. The rectangle $(\Omega ; a, b, c, d)$ is crossed by $\oplus$ in an Ising configuration $\sigma$ if there exists a path of $\oplus$ going from $(a b)$ to $(c d)$ in $\Omega$. We denote this event by $(a b) \stackrel{\oplus}{\longleftrightarrow}(c d)$. We have the following RSW-type estimate on the crossing probability at critical.

Proposition 5.1 ([7], Corollary 1.7). For each $L>0$, there exists $c(L)>0$ such that the following holds: for any topological rectangle $(\Omega ; a, b, c, d)$ with $d_{\Omega}((a b),(c d)) \leq L$,

$$
\mu_{\beta_{c}, \Omega}^{\operatorname{mixed}}[(a b) \stackrel{\oplus}{\longleftrightarrow}(c d)] \geq c(L)
$$

where the boundary conditions are free on $(a b) \cup(c d)$ and $\ominus$ on $(b c) \cup(d a)$.

As a consequence of Proposition 5.1, we have the following spatial mixing property at criticality.

COROLLARY 5.2. There exists $\alpha>0$ such that for any $2 k \leq n$, for any event $\mathcal{A}$ depending only on edges in $\Lambda_{k}$, and for any boundary conditions $\tau, \xi$, we have

$$
\left|\mu_{\beta_{c}, \Lambda_{n}}^{\tau}[\mathcal{A}]-\mu_{\beta_{c}, \Lambda_{n}}^{\xi}[\mathcal{A}]\right| \leq\left(\frac{k}{n}\right)^{\alpha} \mu_{\beta_{c}, \Lambda_{n}}^{\tau}[\mathcal{A}] .
$$

In particular, this implies that, for any boundary conditions $\tau$, for any $2 k \leq n \leq m$, for any event $\mathcal{A}$ depending only on $\left\{\sigma_{x}, x \in \Lambda_{k}\right\}$, and for any event $\mathcal{B}$ depending only on $\left\{\sigma_{x}, x \in \Lambda_{m} \backslash \Lambda_{n}\right\}$, we have

$$
\left|\mu_{\beta_{c}, \Lambda_{m}}^{\tau}[\mathcal{A} \cap \mathcal{B}]-\mu_{\beta_{c}, \Lambda_{m}}^{\tau}[\mathcal{A}] \mu_{\beta_{c}, \Lambda_{m}}^{\tau}[\mathcal{B}]\right| \leq\left(\frac{k}{n}\right)^{\alpha} \mu_{\beta_{c}, \Lambda_{m}}^{\tau}[\mathcal{A}] \mu_{\beta_{c}, \Lambda_{m}}^{\tau}[\mathcal{B}] .
$$


Fix $n<N$ and consider the annulus $\Lambda_{N} \backslash \Lambda_{n}$. A simple path of $\oplus$ or of $\ominus$ connecting $\partial \Lambda_{n}$ to $\partial \Lambda_{N}$ is called an $\mathrm{arm}$. Fix an integer $j \geq 1$ and $\omega=\left(\omega_{1}, \ldots, \omega_{j}\right) \in$ $\{\ominus, \oplus\}^{j}$. For $n<N$, define $\mathcal{A}_{\omega}(n, N)$ to be the event that there are $j$ disjoint arms $\left(\gamma_{k}\right)_{1 \leq k \leq j}$ connecting $\partial \Lambda_{n}$ to $\partial \Lambda_{N}$ in the annulus $\Lambda_{N} \backslash \Lambda_{n}$ which are of types $\left(\omega_{k}\right)_{1 \leq k \leq j}$, where we identify two sequences $\omega$ and $\omega^{\prime}$ if they are the same up to cyclic permutation and the arms are indexed in clockwise order. For each $j \geq 1$, there exists a smallest integer $n_{0}(j)$ such that, for all $N \geq n_{0}(j)$, we have $\mathcal{A}_{\omega}\left(n_{0}(j), N\right) \neq \varnothing$.

Proposition 5.3. Assume that $\omega$ is alternating with even length. For all $n_{0}(j) \leq n_{1}<n_{2}<n_{3} \leq m / 2$, and for all boundary conditions $\tau$, we have

$$
\mu_{\beta_{c}, \Lambda_{m}}^{\tau}\left[\mathcal{A}_{\omega}\left(n_{1}, n_{3}\right)\right] \asymp \mu_{\beta_{c}, \Lambda_{m}}^{\tau}\left[\mathcal{A}_{\omega}\left(n_{1}, n_{2}\right)\right] \mu_{\beta_{c}, \Lambda_{m}}^{\tau}\left[\mathcal{A}_{\omega}\left(n_{2}, n_{3}\right)\right],
$$

where the constants in $\asymp$ are uniform over $n_{1}, n_{2}, n_{3}, m$ and $\tau$.

Proposition 5.3 is called the quasi-multiplicativity. We will introduce several auxiliary subevents of $\mathcal{A}_{\omega}(n, N)$ which are both important for the proof of Proposition 5.3 and also important for us to derive the arm exponents of the Ising model. Fix $\omega=\left(\omega_{1}, \ldots, \omega_{j}\right) \in\{\ominus, \oplus\}^{j}$. Fix some $\delta>0$ small. Suppose $Q=[-1,1]^{2}$ is the unit square. A landing sequence $\left(I_{k}\right)_{1 \leq k \leq j}$ is a sequence of disjoint subintervals on $\partial Q$ in clockwise order. We denote by $z\left(I_{k}\right)$ the center of $I_{k}$. We say $\left(I_{k}\right)_{1 \leq k \leq j}$ is $\delta$-separated if:

- the intervals are at distance at least $2 \delta$ from each other, and they are at distance at least $2 \delta$ from the four corners of $\partial Q$

- for each $I_{k}$, the length of $I_{k}$ is at least $2 \delta$.

We say that two sets are $\omega_{k}$-connected if there is a path of type $\omega_{k}$ connecting them. Fix two $\delta$-separated landing sequences $\left(I_{k}\right)_{1 \leq k \leq j}$ and $\left(I_{k}^{\prime}\right)_{1 \leq k \leq j}$. We say that the arms $\left(\gamma_{k}\right)_{1 \leq k \leq j}$ are $\delta$-well-separated with landing sequence $\left(I_{k}\right)_{1 \leq k \leq j}$ on $\partial \Lambda_{n}$ and landing sequence $\left(I_{k}^{\prime}\right)_{1 \leq k \leq j}$ on $\partial \Lambda_{N}$ if:

- for each $k$, the arm $\gamma_{k}$ connects $n I_{k}$ to $N I_{k}^{\prime}$;

- for each $k$, the arm $\gamma_{k}$ can be $\omega_{k}$-connected to distance $\delta n$ of $\partial \Lambda_{n}$ inside $\Lambda_{\delta n}\left(z\left(I_{k}\right)\right)$

- for each $k$, the arm $\gamma_{k}$ can be $\omega_{k}$-connected to distance $\delta N$ of $\partial \Lambda_{N}$ inside $\Lambda_{\delta N}\left(z\left(I_{k}^{\prime}\right)\right)$.

We denote this event by $\mathcal{A}_{\omega}^{I / I^{\prime}}(n, N)$.

LEMMA 5.4. Fix $j \geq 1$ and $\delta>0$ and two $\delta$-separated landing sequences $\left(I_{k}\right)_{1 \leq k \leq j}$ and $\left(I_{k}^{\prime}\right)_{1 \leq k \leq j}$. Assume that $\omega$ is alternating with length $2 j$. For all $n<N \leq m / 2$ such that $\mathcal{A}_{\omega}^{I / I^{\prime}}(n, N)$ is not empty, and for all boundary conditions 
$\tau$, we have

$$
\mu_{\beta_{c}, \Lambda_{m}}^{\tau}\left[\mathcal{A}_{\omega}^{I / I^{\prime}}(n, N)\right] \asymp \mu_{\beta_{c}, \Lambda_{m}}^{\tau}\left[\mathcal{A}_{\omega}(n, N)\right],
$$

where the constants in $\asymp$ depend only on $\delta$.

We have similar results for the boundary arm events. Denote by

$$
\Lambda_{n}^{+}(x)=[-n, n] \times[0, n]+x, \quad \Lambda_{n}^{+}=\Lambda_{n}^{+}(0) .
$$

We consider the arm events in the semi-annulus $\Lambda_{N}^{+} \backslash \Lambda_{n}^{+}$and extend the definition of arm events and arm events with landing sequences in the obvious way, and denote them by $\mathcal{A}_{\omega}^{+}(n, N)$ and $\mathcal{A}_{\omega}^{+, I / I}(n, N)$.

We need to restrict to the cases that the arms together with the boundary conditions are alternating. Precisely, in the statements of Proposition 5.5 and Lemma 5.6, we restrict to the cases where the arm patterns and the boundary conditions are listed in Theorem 1.1.

PROPOSITION 5.5. For all $n_{0}^{+}(j) \leq n_{1}<n_{2}<n_{3} \leq m / 2$, we have

$$
\mu_{\beta_{c}, \Lambda_{m}^{+}}^{\tau}\left[\mathcal{A}_{\omega}^{+}\left(n_{1}, n_{3}\right)\right] \asymp \mu_{\beta_{c}, \Lambda_{m}^{+}}^{\tau}\left[\mathcal{A}_{\omega}^{+}\left(n_{1}, n_{2}\right)\right] \mu_{\beta_{c}, \Lambda_{m}^{+}}^{\tau}\left[\mathcal{A}_{\omega}^{+}\left(n_{2}, n_{3}\right)\right],
$$

where the constants in $\asymp$ are uniform over $n_{1}, n_{2}, n_{3}$ and $m$.

LEMMA 5.6. Fix $j \geq 1, \delta>0$ and two $\delta$-separated landing sequences $\left(I_{k}\right)_{1 \leq k \leq j}$ and $\left(I_{k}^{\prime}\right)_{1 \leq k \leq j}$. For all $n<N \leq m / 2$ such that $\mathcal{A}_{\omega}^{+, I / I^{\prime}}(n, N)$ is not empty, we have

$$
\mu_{\beta_{c}, \Lambda_{m}^{+}}^{\tau}\left[\mathcal{A}_{\omega}^{+, I / I^{\prime}}(n, N)\right] \asymp \mu_{\beta_{c}, \Lambda_{m}^{+}}^{\tau}\left[\mathcal{A}_{\omega}^{+}(n, N)\right],
$$

where the constants in $\asymp$ depend only on $\delta$.

We do not give the proof of the quasi-multiplicativity in this paper, because the proof is exactly the same as the proof of the quasi-multiplicativity for the FK-Ising model proved in [7].

5.3. Proofs of Theorems 1.1 and 1.2. In this section, we first define the interfaces of the Ising model; then explain the convergence results on the interfaces; and finally, complete the proof of Theorems 1.1 and 1.2.

The dual square lattice $\left(\mathbb{Z}^{2}\right)^{*}$ is the dual graph of $\mathbb{Z}^{2}$. The vertex set is $(1 / 2,1 / 2)+\mathbb{Z}^{2}$ and the edges are given by nearest neighbors. The vertices and edges of $\left(\mathbb{Z}^{2}\right)^{*}$ are called dual-vertices and dual-edges. In particular, for each edge $e$ of $\mathbb{Z}^{2}$, it is associated to a dual edge, denoted by $e^{*}$. The dual edge $e^{*} \operatorname{crosses} e$ in the middle. For a finite subgraph $G$, we define $G^{*}$ to be the subgraph of $\left(\mathbb{Z}^{2}\right)^{*}$ with edge-set $E\left(G^{*}\right)=\left\{e^{*}: e \in E(G)\right\}$ and vertex set given by the end-points of these dual-edges. The medial lattice $\left(\mathbb{Z}^{2}\right)^{\diamond}$ is the graph with the centers of edges of 


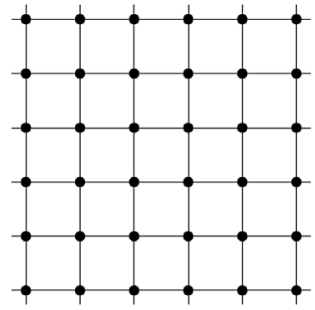

(a) The square lattice.

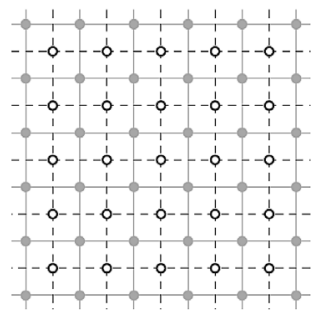

(b) The dual lattice.

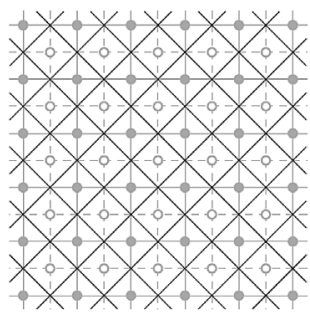

(c) The medial lattice.

FIG. 3. The lattices.

$\mathbb{Z}^{2}$ as vertex set, and edges connecting nearest vertices. This lattice is a rotated and rescaled version of $\mathbb{Z}^{2}$; see Figure 3 . The vertices and edges of $\left(\mathbb{Z}^{2}\right)^{\diamond}$ are called medial-vertices and medial-edges. We identify the faces of $\left(\mathbb{Z}^{2}\right)^{\diamond}$ with the vertices of $\mathbb{Z}^{2}$ and $\left(\mathbb{Z}^{2}\right)^{*}$. A face of $\left(\mathbb{Z}^{2}\right)^{\diamond}$ is said to be black if it corresponds to a vertex of $\mathbb{Z}^{2}$ and white if it corresponds to a vertex of $\left(\mathbb{Z}^{2}\right)^{*}$.

For $u>0$, we consider the rescaled square lattice $u \mathbb{Z}^{2}$. The definitions of dual lattice, medial lattice and Dobrushin domains extend to this context, and they will be denoted by $\left(\Omega_{u} ; a_{u}, b_{u}\right),\left(\Omega_{u}^{*} ; a_{u}^{*}, b_{u}^{*}\right),\left(\Omega_{u}^{\diamond} ; a_{u}^{\diamond}, b_{u}^{\diamond}\right)$, respectively. Consider the critical Ising model on $\left(\Omega_{u}^{*} ; a_{u}^{*}, b_{u}^{*}\right)$. The boundary $\partial \Omega_{u}^{*}$ is divided into two parts $\left(a_{u}^{*} b_{u}^{*}\right)$ and $\left(b_{u}^{*} a_{u}^{*}\right)$. We fix the boundary conditions to be $\ominus$ on $\left(b_{u}^{*} a_{u}^{*}\right)$ and $\oplus$ on $\left(a_{u}^{*} b_{u}^{*}\right)$, or $\ominus$ on $\left(b_{u}^{*} a_{u}^{*}\right)$ and free on $\left(a_{u}^{*} b_{u}^{*}\right)$. Define the interface as follows. It starts from $a_{u}^{\diamond}$, lies on the primal lattice and turns at every vertex of $\Omega_{u}$ is such a way that it has always dual vertices with spin $\ominus$ on its left and $\oplus$ on its right. If there is an indetermination when arriving at a vertex (this may happen on the square lattice), turn left; see Figure 4.

Let $(\Omega ; a, b)$ be a simply connected domain with two marked points on its boundary. Consider a sequence of Dobrushin domains $\left(\Omega_{u} ; a_{u}, b_{u}\right)$. We say that

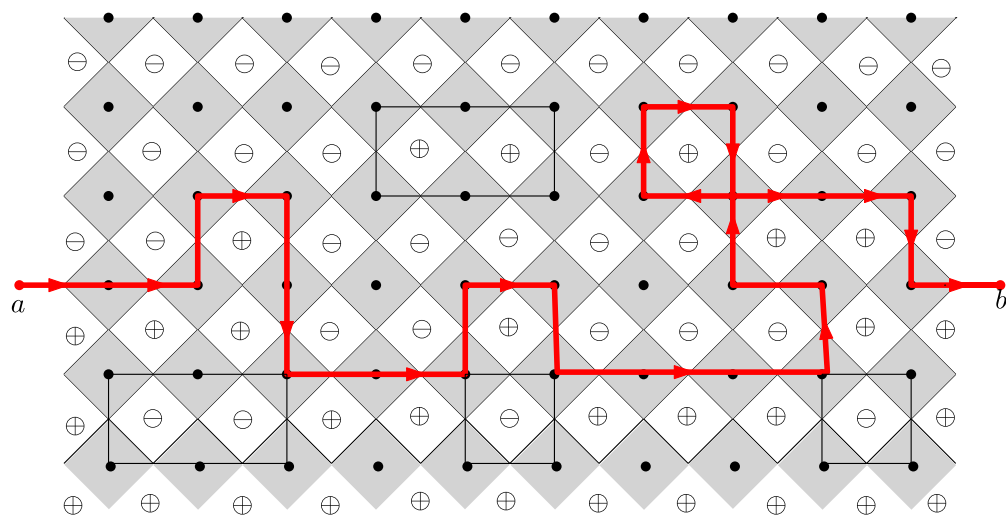

FIG. 4. The Ising interface. 
$\left(\Omega_{u} ; a_{u}, b_{u}\right)$ converges to $(\Omega ; a, b)$ in the Carathéodory sense if $f_{u} \rightarrow f$ on any compact subset $K \subset \mathbb{H}$, where $f_{u}$ (resp., $f$ ) is the unique conformal map from $\mathbb{H}$ to $\Omega_{u}$ (resp., $\Omega$ ) satisfying $f_{u}(0)=a_{u}, f_{u}(\infty)=b_{u}$ and $f_{u}^{\prime}(\infty)=1$ [resp., $\left.f(0)=a, f(\infty)=b, f^{\prime}(\infty)=1\right]$.

Let $X$ be the set of continuous parameterized curves and $d$ be the distance on $X$ defined for $\eta_{1}: I \rightarrow \mathbb{C}$ and $\eta_{2}: J \rightarrow \mathbb{C}$ by

$$
d\left(\eta_{1}, \eta_{2}\right)=\inf _{\varphi_{1}:[0,1] \rightarrow I, \varphi_{2}:[0,1] \rightarrow J} \sup _{t \in[0,1]}\left|\eta_{1}\left(\varphi_{1}(t)\right)-\eta_{2}\left(\varphi_{2}(t)\right)\right|,
$$

where the infimum is over increasing bijective functions $\varphi_{1}, \varphi_{2}$. Note that $I$ and $J$ can be equal to $\mathbb{R}_{+} \cup\{\infty\}$. The topology on $(X, d)$ gives rise to a notion of weak convergence for random curves on $X$.

THEOREM 5.7 ([8]). Let $\left(\Omega_{u}^{\diamond} ; a_{u}^{\diamond}, b_{u}^{\diamond}\right)$ be a family of Dobrushin domains converging to a Dobrushin domain $(\Omega ; a, b)$ in the Carathéodory sense. The interface of the critical Ising model in $\left(\Omega_{u}^{*} ; a_{u}^{*}, b_{u}^{*}\right)$ with $(\ominus \oplus)$ boundary conditions converges weakly to $\mathrm{SLE}_{3}$ as $u \rightarrow 0$.

THEOREM 5.8. Let $\left(\Omega_{u}^{\diamond} ; a_{u}^{\diamond}, b_{u}^{\diamond}\right)$ be a family of Dobrushin domains converging to a Dobrushin domain $(\Omega ; a, b)$ in the Carathéodory sense. The interface of the critical Ising model in $\left(\Omega_{u}^{*} ; a_{u}^{*}, b_{u}^{*}\right)$ with ( $\ominus$ free) boundary conditions converges weakly to $\operatorname{SLE}_{3}(-3 / 2)$ as $u \rightarrow 0$.

PROOF. It is proved in $[5,12]$ that the interface with (free free) boundary conditions converges weakly to $\operatorname{SLE}_{3}(-3 / 2 ;-3 / 2)$ as $u \rightarrow 0$. The same proof works here.

ProOF OF THEOREM 1.2. We only give the proof for $\alpha_{4}$ and the other cases can be proved similarly. Consider $\Lambda_{m}$ with two boundary points $a_{m}=(-m, 0)$ and $b_{m}=(m, 0)$. Fix $(\ominus \oplus)$ boundary conditions: the vertices along $\partial \Lambda_{m}$ from $b_{m}$ to $a_{m}$ (counterclockwise) are $\oplus$ and the vertices from $a_{m}$ to $b_{m}$ are $\ominus$. Since we fix $\beta=\beta_{c}$ and the boundary conditions, and $\omega=(\oplus \ominus \oplus \ominus)$, we eliminate them from the notation. We will prove that, for $n<N \leq m / 2$,

$$
\mu_{\Lambda_{m}}[\mathcal{A}(n, N)]=N^{-\alpha_{4}+o(1)} \quad \text { as } N \rightarrow \infty .
$$

Fix the landing sequence $I=\left(I_{1}, I_{2}, I_{3}, I_{4}\right)$ where $I_{1}=[-1 / 2,1 / 2] \times\{-1\}$, $I_{2}=\{-1\} \times[-1 / 2,1 / 2], I_{3}=[-1 / 2,1 / 2] \times\{1\}$ and $I_{4}=\{1\} \times[-1 / 2,1 / 2]$. Recall that $\mathcal{A}^{I / I}(n, N)$ is the $1 / 8$-well-separated arm event with the landing sequence $n I$ on $\partial \Lambda_{n}$ and $N I$ on $\partial \Lambda_{N}$. The four arms in $\mathcal{A}(n, N)$ are denoted by $\left(\gamma_{1}, \gamma_{2}, \gamma_{3}, \gamma_{4}\right)$ where $\gamma_{1}$ and $\gamma_{3}$ are $\oplus$ and $\gamma_{2}$ and $\gamma_{4}$ are $\ominus$. Consider the critical Ising model in $\Lambda_{2 N}$. Let $R_{1}$ to be the rectangle [ $\left.-3 N / 4,3 N / 4\right] \times[-2 N,-N]$, and define $\mathcal{C}_{1}^{\oplus}$ to be the event that $\gamma_{1}$ is connected by path of $\oplus$ in $R_{1}$ to the bottom of $R_{1}$. Let $R_{2}$ to be the rectangle [ $\left.-9 N / 8,-N\right] \times[-N / 2,2 N]$, and define $\mathcal{C}_{2}^{\ominus}$ to 


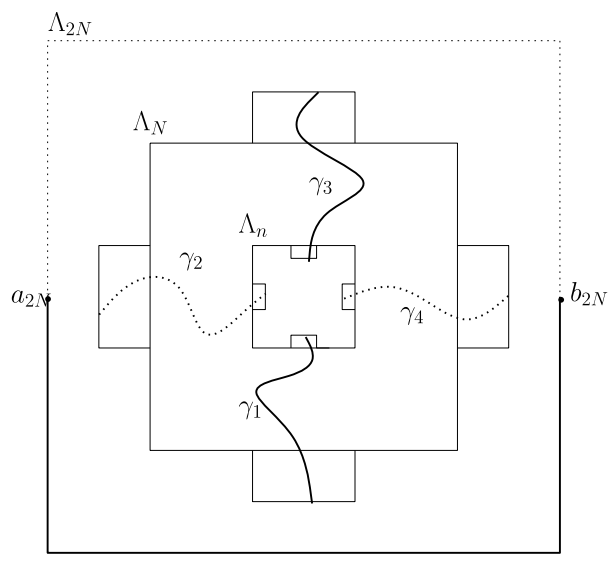

(a) $\mathcal{A}^{I / I}(n, N)$ is the well-separated arm event.

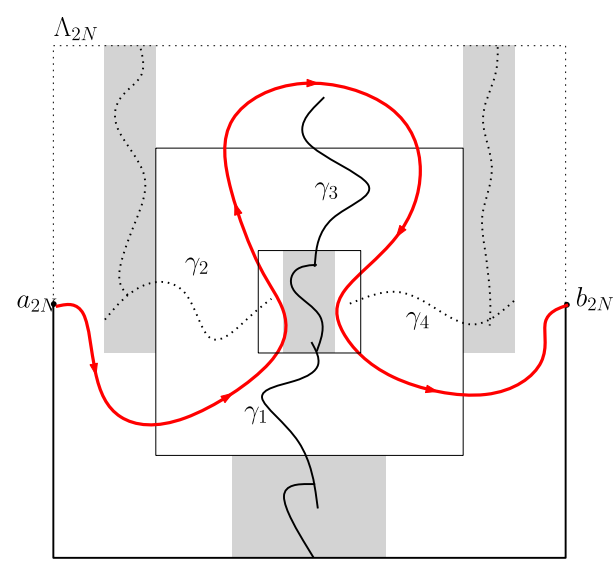

(b) The four gray parts are $R_{1}$ to $R_{4}$ respectively.

FIG. 5. The explanation of the proof of Theorem 1.2.

be the event that $\gamma_{2}$ is connected by path of $\ominus$ in $R_{2}$ to the top of $R_{2}$. Let $R_{3}$ be the rectangle $[-3 n / 4,3 n / 4] \times[-n, n]$, and define $\mathcal{C}_{3}^{\oplus}$ to be the event that $\gamma_{3}$ is connected to $\gamma_{1}$ by path of $\oplus$ in $R_{3}$. Let $R_{4}$ be the rectangle $[N, 9 N / 8] \times[-N / 2,2 N]$, and define $\mathcal{C}_{4}^{\ominus}$ to be the event that $\gamma_{4}$ is connected by path of $\ominus$ in $R_{4}$ to the top of $R_{4}$; see Figure 5.

By (5.1), Proposition 5.1 and Corollary 5.2, we deduce

$$
\mu_{\Lambda_{2 N}}\left[\mathcal{A}^{I / I}(n, N)\right] \asymp \mu_{\Lambda_{2 N}}\left[\mathcal{A}^{I / I}(n, N) \cap \mathcal{C}_{1}^{\oplus} \cap \mathcal{C}_{2}^{\ominus} \cap \mathcal{C}_{3}^{\oplus} \cap \mathcal{C}_{4}^{\ominus}\right],
$$

where the constants in $\asymp$ are uniform over $n, N$.

Let $\mathbb{P}_{N}$ be the probability measure $\mu_{\Lambda_{2 N}}$ where the square lattice is scaled by $1 / N$ and let $\mathbb{P}_{\infty}$ be the law of $\mathrm{SLE}_{3}$ in $[-2,2] \times[-2,2]$ from $(-2,0)$ to $(2,0)$. On the event $\mathcal{A}^{I / I}(n, N) \cap \mathcal{C}_{1}^{\oplus} \cap \mathcal{C}_{2}^{\ominus} \cap \mathcal{C}_{3}^{\oplus} \cap \mathcal{C}_{4}^{\ominus}$, consider the interface $\eta$ from $a_{2 N}$ to $b_{2 N}$. Let $\tau_{1}$ be the first time that $\eta$ hits $\partial \Lambda_{n}$. The event $\mathcal{C}_{1}^{\oplus} \cap \mathcal{C}_{2}^{\ominus}$ guarantees that $\eta\left[0, \tau_{1}\right]$ is bounded away from the target $b_{2 N}$. The event $\mathcal{C}_{3}^{\oplus}$ guarantees that, after $\tau_{1}$, the path $\eta$ hits the neighborhood of $(0,2 N)$ at some time $\sigma_{1}$. The event $\mathcal{C}_{4}^{\ominus}$ guarantees that, after $\sigma_{1}$, the path $\eta$ hits $\partial \Lambda_{n}$ again. Therefore, by (4.1), we have for $\varepsilon>0$,

$$
\begin{gathered}
\limsup _{N \rightarrow \infty} \mathbb{P}_{N}\left[\mathcal{A}^{I / I}(\varepsilon N, N) \cap \mathcal{C}_{1}^{\oplus} \cap \mathcal{C}_{2}^{\ominus} \cap \mathcal{C}_{3}^{\oplus} \cap \mathcal{C}_{4}^{\ominus}\right] \\
\leq \varepsilon^{\alpha_{4}+o(1)} \leq \liminf _{N \rightarrow \infty} \mathbb{P}_{N}[\mathcal{A}(\varepsilon N, N)] .
\end{gathered}
$$

Combining with Lemma 5.4 and (5.3), we have

$$
\liminf _{N \rightarrow \infty} \mathbb{P}_{N}[\mathcal{A}(\varepsilon N, N)] \asymp \limsup _{N \rightarrow \infty} \mathbb{P}_{N}[\mathcal{A}(\varepsilon N, N)] \asymp \varepsilon^{\alpha_{4}+o(1)} .
$$


By Corollary 5.2, we know that

$$
\liminf _{N \rightarrow \infty} \mu_{\Lambda_{m}}[\mathcal{A}(\varepsilon N, N)] \asymp \limsup _{N \rightarrow \infty} \mu_{\Lambda_{m}}[\mathcal{A}(\varepsilon N, N)] \asymp \varepsilon^{\alpha_{4}+o(1)},
$$

where the constants in $\asymp$ are uniform over $\varepsilon$ and $m \geq 2 N$.

Suppose $N=n \varepsilon^{-K}$ for some integer $K$. By Proposition 5.3, for $m \geq 2 N$, we have

$$
\mu_{\Lambda_{m}}[\mathcal{A}(n, N)] \leq C^{K} \prod_{j=1}^{K} \mu_{\Lambda_{m}}\left[\mathcal{A}\left(n \varepsilon^{-j+1}, n \varepsilon^{-j}\right)\right]
$$

where $C$ is some universal constant. Thus

$$
\frac{\log \mu_{\Lambda_{m}}[\mathcal{A}(n, N)]}{\log N} \leq \frac{K \log C}{\log N}+\frac{1}{\log N} \sum_{j=1}^{K} \log \mu_{\Lambda_{m}}\left[\mathcal{A}\left(n \varepsilon^{-j-1}, n \varepsilon^{-j}\right)\right]
$$

By (5.4), we have

$$
\limsup _{j \rightarrow \infty} \mu_{\Lambda_{m}}\left[\mathcal{A}\left(n \varepsilon^{-j-1}, n \varepsilon^{-j}\right)\right] \asymp \varepsilon^{\alpha_{4}+o(1)} .
$$

Therefore,

$$
\limsup _{K \rightarrow \infty} \frac{\log \mu_{\Lambda_{m}}[\mathcal{A}(n, N)]}{\log N} \leq \frac{\tilde{C}}{\log (1 / \varepsilon)}-\alpha_{4}
$$

where $\tilde{C}$ is some universal constant. Let $\varepsilon \rightarrow 0$, we have

$$
\limsup _{N \rightarrow \infty} \frac{\log \mu_{\Lambda_{m}}[\mathcal{A}(n, N)]}{\log N} \leq-\alpha_{4} .
$$

We deduce the lower bound similarly:

$$
\liminf _{N \rightarrow \infty} \frac{\log \mu_{\Lambda_{m}}[\mathcal{A}(n, N)]}{\log N} \geq-\alpha_{4} .
$$

These imply (5.2) and complete the proof.

To prove Theorem 1.1, we will show the proof for $\gamma_{2 j-1}^{+}$, and the results for $\alpha_{2 j-1}^{+}, \gamma_{2 j}^{+}$can be proved similarly; and we will show the proof for $\beta_{2 j}^{+}$, and the results for $\alpha_{2 j}^{+}, \beta_{2 j-1}^{+}$can be proved similarly.

ProOf OF THEOREM 1.1-(1.5). We will prove the conclusion for $\gamma_{3}^{+}$and the other cases can be proved similarly. Consider $\Lambda_{m}^{+}$with two boundary points $a_{m}=$ $(-m, m / 2)$ and $b_{m}=(m, m / 2)$. Fix ( $\ominus$ free) boundary conditions: the vertices along $\partial \Lambda_{m}$ from $b_{m}$ to $a_{m}$ (counterclockwise) are free and the vertices from $a_{m}$ to 


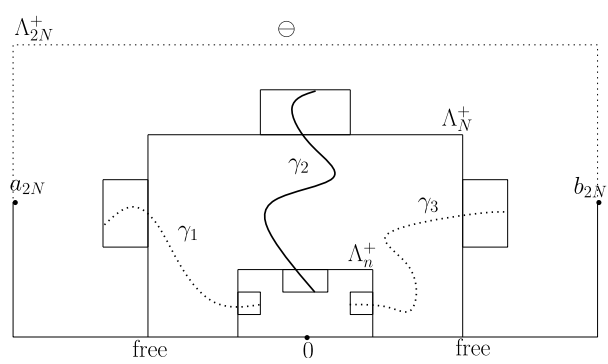

(a) $\mathcal{A}^{+}, I / I(n, N)$ is the well-separated arm event.

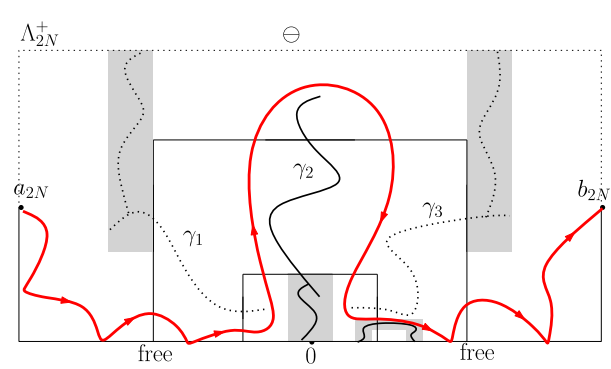

(b) The four gray parts are $R_{1}$ to $R_{4}$ respectively.

FIG. 6. The explanation of the proof of (1.5).

$b_{m}$ are $\ominus$. Since we fix $\beta=\beta_{c}$ and the boundary conditions, and $\omega=(\ominus \ominus \ominus)$, we eliminate them from the notation. We will prove that, for $n<N \leq m / 2$,

$$
\mu_{\Lambda_{m}^{+}}\left[\mathcal{A}^{+}(n, N)\right]=N^{-\gamma_{3}^{+}+o(1)} \quad \text { as } N \rightarrow \infty .
$$

Fix the landing sequence $I=\left(I_{1}, I_{2}, I_{3}\right)$ where $I_{1}=\{-1\} \times[1 / 2,3 / 4], I_{2}=$ $[-1 / 2,1 / 2] \times\{1\}$ and $I_{3}=\{1\} \times[1 / 2,3 / 4]$. Recall that $\mathcal{A}^{+, I / I}(n, N)$ is the $1 / 8-$ well-separated arm event with the landing sequence $n I$ on $\partial \Lambda_{n}^{+}$and $N I$ on $\partial \Lambda_{N}^{+}$. The three arms in $\mathcal{A}^{+, I / I}(n, N)$ are denoted by $\left(\gamma_{1}, \gamma_{2}, \gamma_{3}\right)$ where $\gamma_{1}$ and $\gamma_{3}$ are $\ominus$ and $\gamma_{2}$ is $\oplus$. Consider the critical Ising model in $\Lambda_{2 N}^{+}$. Let $R_{1}$ be the rectangle $[-9 N / 8,-N] \times[N / 2, N]$ and define $\mathcal{C}_{1}^{\ominus}$ to be the event that $\gamma_{1}$ is connected by path of $\ominus$ in $R_{1}$ to the top of $R_{1}$. Let $R_{2}$ be the rectangle [ $\left.-3 n / 4,3 n / 4\right] \times[0, n]$ and define $\mathcal{C}_{2}^{\oplus}$ to be the event that $\gamma_{2}$ is connected by path of $\oplus$ in $R_{2}$ to the bottom of $R_{2}$. Let $R_{3}$ be the rectangle [N,9N/8] $\times[N / 2, N]$ and define $\mathcal{C}_{3}^{\ominus}$ to be the event that $\gamma_{3}$ is connected by path of $\ominus$ in $R_{3}$ to the top of $R_{3}$. For $\delta>0$, let $R_{4}$ be the semi-annulus $[3 n / 4,4 n] \times[0, n / 4] \backslash[n, 3 n] \times[0, \delta n]$ and define $\mathcal{C}_{4}^{\oplus}(\delta)$ to be the event that there is a path of $\oplus$ in $R_{4}$ connecting the left bottom to the right bottom.

By (5.1), Proposition 5.1 and Corollary 5.2, we can prove, for $\delta>0$ small enough,

$$
\mu_{\Lambda_{2 N}}\left[\mathcal{A}^{+, I / I}(n, N)\right] \asymp \mu_{\Lambda_{2 N}}\left[\mathcal{A}^{+, I / I}(n, N) \cap \mathcal{C}_{1}^{\ominus} \cap \mathcal{C}_{2}^{\oplus} \cap \mathcal{C}_{3}^{\ominus} \cap \mathcal{C}_{4}^{\oplus}(\delta)\right],
$$

where the constants in $\asymp$ are uniform over $n, N$.

Let $\mathbb{P}_{N}$ be the probability measure $\mu_{\Lambda_{2 N}}$ where the square lattice is scaled by $1 / N$ and let $\mathbb{P}_{\infty}$ be the law of $\operatorname{SLE}_{3}(-3 / 2)$ in $[-2,2] \times[0,2]$ from $(-2,1)$ to $(2,1)$. On the event $\mathcal{A}^{+, I / I}(n, N) \cap \mathcal{C}_{1}^{\ominus} \cap \mathcal{C}_{2}^{\oplus} \cap \mathcal{C}_{3}^{\ominus} \cap \mathcal{C}_{4}^{\oplus}(\delta)$, consider the interface $\eta$ from $a_{2 N}$ to $b_{2 N}$. Let $\tau_{1}$ be the first time that $\eta$ hits $\partial \Lambda_{n}$. The event $\mathcal{C}_{1}^{\ominus}$ guarantees that $\eta\left[0, \tau_{1}\right]$ is bounded away from the target $b_{2 N}$. The event $\mathcal{C}_{4}^{\oplus}(\delta)$ guarantees that $\eta\left[0, \tau_{1}\right]$ is bounded away from the segment $[n, 3 n]$. The event $\mathcal{C}_{2}^{\oplus}$ guarantees that, after $\tau_{1}$, the interface $\eta$ hits the neighborhood of the point $(0, N)$ at some time $\sigma_{1}$. 
The event $\mathcal{C}_{3}^{\ominus}$ guarantees that, after $\sigma_{1}$, the interface $\eta$ hits $\partial \Lambda_{n}$ again; see Figure 6 . Therefore, by (3.11), we have for $\varepsilon>0$,

$$
\begin{gathered}
\limsup _{N \rightarrow \infty} \mathbb{P}_{N}\left[\mathcal{A}^{+, I / I}(\varepsilon N, N) \cap \mathcal{C}_{1}^{\ominus} \cap \mathcal{C}_{2}^{\oplus} \cap \mathcal{C}_{3}^{\ominus} \cap \mathcal{C}_{4}^{\oplus}(\delta)\right] \\
\lesssim \varepsilon^{\gamma_{3}^{+}} \leq \liminf _{N \rightarrow \infty} \mathbb{P}_{N}\left[\mathcal{A}^{+}(\varepsilon N, N)\right] .
\end{gathered}
$$

Now we can repeat the same proof of Theorem 1.2 to obtain (5.5).

Proof of Theorem 1.1-(1.4). We will prove the conclusion for $\beta_{2}^{+}$and the other cases can be proved similarly. Consider $\Lambda_{m}^{+}$with two boundary points $a_{m}=(0,0)$ and $b_{m}=(0, m)$. Fix ( $\ominus$ free) boundary conditions: the vertices along $\partial \Lambda_{m}$ from $b_{m}$ to $a_{m}$ (counterclockwise) are free and the vertices from $a_{m}$ to $b_{m}$ are $\ominus$. Since we fix $\beta=\beta_{c}$ and the boundary conditions, and $\omega=(\oplus \ominus)$, we eliminate them from the notation. We will prove that, for $n<N \leq m / 2$,

$$
\mu_{\Lambda_{m}^{+}}\left[\mathcal{A}^{+}(n, N)\right]=N^{-\beta_{2}^{+}+o(1)} \quad \text { as } N \rightarrow \infty .
$$

Fix the landing sequence $I=\left(I_{1}, I_{2}\right)$ where $I_{1}=\{-1\} \times[1 / 2,3 / 4]$ and $I_{2}=[-1 / 2,1 / 2] \times\{1\}$. Recall that $\mathcal{A}^{+, I / I}(n, N)$ is the $1 / 8$-well-separated arm event with the landing sequence $n I$ on $\partial \Lambda_{n}^{+}$and $N I$ on $\partial \Lambda_{N}^{+}$. The two arms in $\mathcal{A}^{+, I / I}(n, N)$ are denoted by $\left(\gamma_{1}, \gamma_{2}\right)$ where $\gamma_{1}$ is $\oplus$ and $\gamma_{2}$ is $\ominus$. Consider the critical Ising model in $\Lambda_{2 N}^{+}$. Let $R_{1}$ be the tube $[-n, 3 n / 4] \times[0,3 n / 4] \backslash[-n, n / 2] \times$ [0,n/4] and define $\mathcal{C}_{1}^{\oplus}$ to be the event that $\gamma_{1}$ is connected by path of $\oplus$ in $R_{1}$ to the bottom of $R_{1}$. Let $R_{2}$ be the rectangle [ $\left.-N, N / 2\right] \times[N / 2,5 N / 8]$ and define $\mathcal{C}_{2}^{\ominus}$ to be the event that $\gamma_{2}$ is connected by path of $\ominus$ in $R_{2}$ to the left-hand side of $R_{2}$.

By (5.1), Proposition 5.1 and Corollary 5.2, we can prove, for $\delta>0$ small enough,

$$
\mu_{\Lambda_{2 N}}\left[\mathcal{A}^{+, I / I}(n, N)\right] \asymp \mu_{\Lambda_{2 N}}\left[\mathcal{A}^{+, I / I}(n, N) \cap \mathcal{C}_{1}^{\oplus} \cap \mathcal{C}_{2}^{\ominus}\right],
$$

where the constants in $\asymp$ are uniform over $n, N$.

Let $\mathbb{P}_{N}$ be the probability measure $\mu_{\Lambda_{2 N}}$ where the square lattice is scaled by $1 / N$ and let $\mathbb{P}_{\infty}$ be the law of $\operatorname{SLE}_{3}(-3 / 2)$ in $[-2,2] \times[0,2]$ from $(0,0)$ to $(0,1)$. On the event $\mathcal{A}^{+, I / I}(n, N) \cap \mathcal{C}_{1}^{\oplus} \cap \mathcal{C}_{2}^{\ominus}$, consider the interface $\eta$ from $a_{2 N}$ to $b_{2 N}$, the event guarantees that the interface hits the neighborhood of the point $(-N, N / 2)$, and then comes back to $\Lambda_{n}^{+}$; see Figure 7. Therefore, by (3.4) and (3.6) (taking $\rho=-3 / 2$ ), we have, for $\varepsilon>0$,

$$
\limsup _{N \rightarrow \infty} \mathbb{P}_{N}\left[\mathcal{A}^{+, I / I}(n, N) \cap \mathcal{C}_{1}^{\oplus} \cap \mathcal{C}_{2}^{\ominus}\right] \lesssim \varepsilon^{\beta_{2}^{+}} \leq \liminf _{N \rightarrow \infty} \mathbb{P}_{N}\left[\mathcal{A}^{+}(\varepsilon N, N)\right] .
$$

Now we can repeat the same proof of Theorem 1.2 to obtain (5.7). 


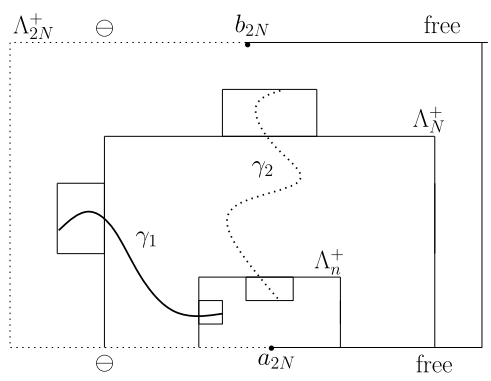

(a) $\mathcal{A}^{+}, I / I(n, N)$ is the well-separated arm event.

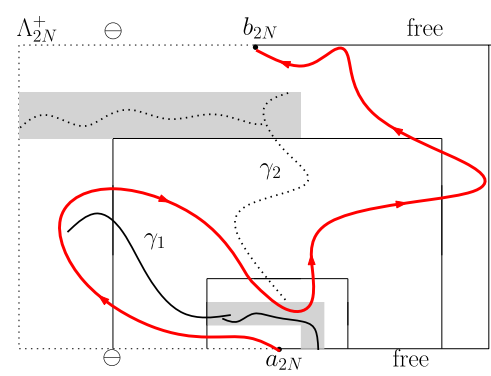

(b) The two gray parts are $R_{1}$ and $R_{2}$ respectively.

FIG. 7. The explanation of the proof of (1.4).

\section{APPENDIX: ONE-POINT ESTIMATE OF THE INTERSECTION OF SLE WITH THE BOUNDARY}

THEOREM A.1. Fix $\kappa>0, \rho^{L}, \rho^{1, R}, \rho^{2, R} \in \mathbb{R}$ such that

$$
\rho^{L}>-2, \quad \rho^{1, R}>(-2) \vee(\kappa / 2-4), \quad \rho^{1, R}+\rho^{2, R}>\kappa / 2-4 .
$$

Suppose $\eta$ is an $\operatorname{SLE}_{\kappa}\left(\rho^{L} ; \rho^{1, R}, \rho^{2, R}\right)$ process with force points $\left(x^{L} ; x^{R}, 1\right)$ where $x^{L} \leq 0$ and $x^{R} \in[0,1)$. Define

$\alpha=\left(\rho^{1, R}+2\right)\left(\rho^{1, R}+\rho^{2, R}+4-\kappa / 2\right) / \kappa, \quad \beta=2\left(\rho^{1, R}+\rho^{2, R}+4-\kappa / 2\right) / \kappa$.

For $\varepsilon \in(0,1 / 2)$ and $r \geq 4$, define $\tau_{\varepsilon}=\inf \left\{t: \eta(t) \in \partial B\left(1, \varepsilon\left(1-x^{R}\right)\right)\right\}$, and $S_{r}=$ $\inf \{t: \eta(t) \in \partial B(0, r)\}$. Then we have

$$
\varepsilon^{\alpha}\left(1-x^{R}\right)^{\beta} \lesssim \mathbb{P}\left[\tau_{\varepsilon} \leq S_{r}\right]=\varepsilon^{\alpha+o(1)},
$$

where the constant in $\lesssim$ is uniform and the $o$ (1) term goes to zero as $\varepsilon \rightarrow 0$ at a rate which depends only on $x^{R}$ and $r$.

COROllary A.2. Fix $\kappa>0, \rho^{L}, \rho^{1, R}, \rho^{2, R} \in \mathbb{R}$ such that

$$
\text { (A.2) } \quad \rho^{L} \in(-2,0], \quad \rho^{1, R}>(-2) \vee(\kappa / 2-4), \quad \rho^{1, R}+\rho^{2, R}>\kappa / 2-4 \text {. }
$$

Assume the same notation as in Theorem A.1. Then we have

$$
\varepsilon^{\alpha}\left(1-x^{R}\right)^{\beta} \lesssim \mathbb{P}\left[\tau_{\varepsilon}<\infty\right]=\varepsilon^{\alpha+o(1)},
$$

where the constant in $\lesssim$ is uniform and the o(1) term goes to zero as $\varepsilon \rightarrow 0$ at a rate which depends only on $x^{R}$.

We also expect that Corollary A.2 holds for all $\rho^{L}>-2$, but we do not have a proof yet for $\rho^{L} \geq 0$. Before proving the theorem, we first summarize the existing 
related results. For standard $\mathrm{SLE}_{\kappa}$ with $\kappa \in(0,8)$, a stronger conclusion is known [3]:

$$
\mathbb{P}[\eta \text { hits } B(1, \varepsilon)] \asymp \varepsilon^{\alpha} \quad \text { where } \alpha=(8-\kappa) / \kappa .
$$

For $\operatorname{SLE}_{\kappa}(\rho)$ with one force point at $x^{R} \in[0,1)$, a stronger conclusion is known ([14], Proposition 5.4):

$$
\mathbb{P}\left[\tau_{\varepsilon}<\infty\right] \asymp \varepsilon^{\alpha}\left(1-x^{R}\right)^{\beta},
$$

where $\alpha=(\rho+2)(\rho+4-\kappa / 2) / \kappa$, and $\beta=2(\rho+4-\kappa / 2) / \kappa$. For $\operatorname{SLE}_{\kappa}\left(\rho^{1, R}\right.$, $\rho^{2, R}$ ) processes, the conclusion in Theorem A.1 is proved in [18], Theorem 3.1. The proof here is simpler than the one in [18].

LEMmA A.3. Assume the same notation as in Theorem A.1. For $\delta \in(0,1 / 4)$ and $r \geq 4$, we have

$$
\varepsilon^{\alpha}\left(1-x^{R}\right)^{\beta} \lesssim \mathbb{P}\left[\tau_{\varepsilon} \leq S_{r}, \operatorname{Im} \eta\left(\tau_{\varepsilon}\right) \geq \delta \varepsilon\left(1-x^{R}\right)\right] \lesssim \varepsilon^{\alpha}\left(1-x^{R}\right)^{\beta} \delta^{-\beta} r^{B},
$$

where $B=0 \vee\left(\beta \rho^{L} / 2\right)$ and the constants in $\lesssim$ are uniform over $\varepsilon, \delta, x^{L}, x^{R}, r$.

Proof. Let $V_{t}^{L}$ be the evolution of $x^{L}$ and $V_{t}^{R}$ be the evolution of $x^{R}$. Set $v=-\beta \kappa \leq 0$ and

$$
\begin{aligned}
M_{t}= & g_{t}^{\prime}(1)^{\nu\left(\nu+2 \rho^{2, R}+4-\kappa\right) /(4 \kappa)}\left(g_{t}(1)-W_{t}\right)^{\nu / \kappa} \\
& \times\left(\frac{g_{t}(1)-V_{t}^{R}}{1-x^{R}}\right)^{v \rho^{1, R} /(2 \kappa)}\left(\frac{g_{t}(1)-V_{t}^{L}}{1-x^{L}}\right)^{\nu \rho^{L} /(2 \kappa)} .
\end{aligned}
$$

By Lemma 2.4, the process $M$ is a local martingale and the law of $\eta$ weighted by $M$ becomes the law of $\operatorname{SLE}_{\kappa}\left(\rho^{L} ; \rho^{1, R}, \rho^{2, R}+v\right)$ with force points $\left(x^{L} ; x^{R}, 1\right)$.

On $E_{\varepsilon}(\delta, r):=\left\{\tau_{\varepsilon} \leq S_{r}, \operatorname{Im} \eta\left(\tau_{\varepsilon}\right) \geq \delta \varepsilon\left(1-x^{R}\right)\right\}$, let us estimate the terms in $M_{t}$ one by one for $t=\tau_{\varepsilon}$. Let $O_{t}$ be the image of the rightmost point of $\eta[0, t] \cap \mathbb{R}$ under $g_{t}$. By the Koebe $1 / 4$ theorem, we have $g_{t}(1)-O_{t} \asymp g_{t}^{\prime}(1) \varepsilon\left(1-x^{R}\right)$.

- Consider the term $g_{t}(1)-W_{t}$. Since $\operatorname{Im} \eta(t) \geq \delta \varepsilon\left(1-x^{R}\right)$, combining with [18], Lemma 3.4, we have

$$
\begin{aligned}
g_{t}^{\prime}(1) \varepsilon\left(1-x^{R}\right) & \asymp g_{t}(1)-O_{t} \leq g_{t}(1)-W_{t} \lesssim\left(g_{t}(1)-O_{t}\right) / \delta \\
& \asymp g_{t}^{\prime}(1) \varepsilon\left(1-x^{R}\right) / \delta .
\end{aligned}
$$

- Consider the term $g_{t}(1)-V_{t}^{R}$. If $x^{R}$ is swallowed by $\eta[0, t]$, then we have $g_{t}(1)-V_{t}^{R}=g_{t}(1)-O_{t} \asymp g_{t}^{\prime}(1) \varepsilon\left(1-x^{R}\right)$. If not, by the Keobe $1 / 4$ theorem, we have $g_{t}(1)-V_{t}^{R} \asymp g_{t}^{\prime}(1) \varepsilon\left(1-x^{R}\right)$. In any case, we have

$$
g_{t}(1)-V_{t}^{R} \asymp g_{t}^{\prime}(1) \varepsilon\left(1-x^{R}\right) \text {. }
$$


- Consider the term $g_{t}(1)-V_{t}^{L}$. Since $g_{t}(1)-V_{t}^{L}$ is increasing in $t$, we have $g_{t}(1)-V_{t}^{L} \geq 1-x^{L}$ for all $t$. Suppose $\mathcal{B}^{y i}$ is a Brownian motion starting from $y i$, from [13], Remark 3.50, we know that

$$
\begin{aligned}
g_{t}(1)- & V_{t}^{L} \\
= & \lim _{y \rightarrow \infty} \pi y \mathbb{P}\left[\mathcal{B}^{y i} \text { exits } \mathbb{H} \backslash \eta[0, t]\right. \text { through the union } \\
& {\left.\left[x^{L}, 0\right] \cup \eta[0, t] \cup[0,1]\right] . }
\end{aligned}
$$

Since $t=\tau_{\varepsilon} \leq S_{r}$, we have

$$
\begin{aligned}
g_{t}(1) & -V_{t}^{L} \\
& \leq \lim _{y \rightarrow \infty} \pi y \mathbb{P}\left[\mathcal{B}^{y i} \text { exits } \mathbb{H} \backslash B(0, r) \text { through the union }\left[x^{L}, 0\right] \cup B(0, r)\right] .
\end{aligned}
$$

If $\left|x^{L}\right| \leq r$, then $g_{t}(1)-V_{t}^{L} \leq 4 r$; if $\left|x^{L}\right| \geq r$, we have $g_{t}(1)-V_{t}^{L} \leq\left|x^{L}\right|+3 r$. Thus we have

$$
1 \leq \frac{g_{t}(1)-V_{t}^{L}}{1-x^{L}} \leq 4 r .
$$

Combining the above three parts, on $E_{\varepsilon}(\delta, r)$, we have

$$
\varepsilon^{-\alpha}\left(1-x^{R}\right)^{-\beta} \delta^{\beta} r^{-B} \lesssim M_{\tau_{\varepsilon}} \lesssim \varepsilon^{-\alpha}\left(1-x^{R}\right)^{-\beta} r^{0 \vee\left(-\beta \rho^{L} / 2\right)}
$$

Therefore, we have the lower bound: $\mathbb{P}\left[E_{\varepsilon}(\delta, r)\right] \geq \mathbb{P}\left[E_{\varepsilon}(4,1 / 4)\right]$ and

$$
\mathbb{P}\left[E_{\varepsilon}(4,1 / 4)\right] \gtrsim \varepsilon^{\alpha}\left(1-x^{R}\right)^{\beta} \mathbb{E}\left[M_{\tau_{\varepsilon}} \mathbb{1}_{E_{\varepsilon}(4,1 / 4)}\right]=\varepsilon^{\alpha}\left(1-x^{R}\right)^{\beta} \mathbb{P}^{*}\left[E_{\varepsilon}^{*}(4,1 / 4)\right],
$$

where $\eta^{*}$ is an $\operatorname{SLE}_{\kappa}\left(\rho^{L} ; \rho^{1, R}, \rho^{2, R}+v\right)$ with force points $\left(x^{L} ; x^{R}, 1\right)$ and $\mathbb{P}^{*}$ is its law, and $E_{\varepsilon}^{*}(r, \delta)$ is defined for $\eta^{*}$. Note that

$$
\rho^{1, R}+\rho^{2, R}+v=\kappa-8-\rho^{1, R}-\rho^{2, R}<\kappa / 2-4 .
$$

Thus $\eta^{*}$ converges to the point 1 at finite time. Let $\phi(z)=z /(1-z)$ be the Mobius transform of $\mathbb{H}$ that sends $(0,1, \infty)$ to $(0, \infty,-1)$ and let $\hat{\eta}$ be the image of $\eta^{*}$ under $\phi$. Then $\hat{\eta}$ is an $\operatorname{SLE}_{\kappa}\left(\rho^{1, R}+\rho^{2, R}+2-\rho^{L}, \rho^{L} ; \rho^{1, R}\right)$ with force points $\left(-1, \phi\left(x^{L}\right) ; \phi\left(x^{R}\right)\right)$. Define $\hat{E}$ to be the event that $\hat{\eta}$ never hits $B(-1,1 / 3)$ and $\hat{\eta}$ exits the ball of radius $1 /\left(\varepsilon\left(1-x^{R}\right)\right)$ through the angle interval $[\pi / 4,3 \pi / 4]$. It is clear that $\mathbb{P}^{*}\left[E_{\varepsilon}^{*}(4,1 / 4)\right] \geq \hat{\mathbb{P}}[\hat{E}] \asymp 1$ (see, for instance, [18], Lemma 2.3), since $\rho^{1, R}+\rho^{2, R}+2>\kappa / 2-2$. This gives the lower bound. For the upper bound, we have

$$
\mathbb{P}\left[E_{\varepsilon}(\delta, r)\right] \lesssim \varepsilon^{\alpha}\left(1-x^{R}\right)^{\beta} \delta^{-\beta} r^{B} \mathbb{E}\left[M_{\tau_{\varepsilon}} \mathbb{1}_{E_{\varepsilon}(\delta, r)}\right] \leq \varepsilon^{\alpha}\left(1-x^{R}\right)^{\beta} \delta^{-\beta} r^{B},
$$

as desired. 
LEMMA A.4. Fix $\kappa>0$ and $\rho^{L}, \rho^{R} \in \mathbb{R}$. Suppose that $x_{n}^{L}\left(\right.$ resp., $\left.x_{n}^{R}\right)$ is a sequence of negative (resp., positive) real numbers converging to $x^{L} \leq 0$ (resp., $\left.x^{R} \geq 0\right)$ as $n \rightarrow \infty$. For each $n$, suppose that $\left(W_{n}, V_{n}^{L}, V_{n}^{R}\right)$ is the driving triple for an $\operatorname{SLE}_{\kappa}\left(\rho^{L} ; \rho^{R}\right)$ process in $\mathbb{H}$ with force points $\left(x_{n}^{L} ; x_{n}^{R}\right)$. Then $\left(W_{n}, V_{n}^{L}, V_{n}^{R}\right)$ converges weakly in law with respect to the local uniform topology to the driving triple $\left(W, V^{L}, V^{R}\right)$ of a $\operatorname{SLE}_{\kappa}\left(\rho^{L} ; \rho^{R}\right)$ with force points $\left(x^{L} ; x^{R}\right)$ as $n \rightarrow \infty$. the same likewise holds in the setting of multiple force points $\operatorname{SLE}_{\kappa}(\underline{\rho})$ processes.

PROOF. Proof of Theorem 2.4 in [17].

LEMMA A.5. Assume (A.1) holds. Suppose that $\eta$ is a $\operatorname{SLE}_{\kappa}\left(\rho^{L} ; \rho^{1, R}, \rho^{2, R}\right)$ with force points $\left(x^{L} ; x^{1, R}, x^{2, R}\right)$ where $x^{L} \leq 0$ and $0 \leq x^{1, R} \leq 1$ and $x^{1, R} \leq$ $x^{2, R}$. Then there exists a function $p(\delta) \rightarrow 0$ as $\delta \rightarrow 0$ which depends only on $\kappa, \rho^{L}, \rho^{1, R}, \rho^{2, R}$ such that

$$
\mathbb{P}[\eta \text { hits } B(1, \delta)] \leq p(\delta) .
$$

We emphasize that $p(\delta)$ is uniform over $x^{L} \leq 0 \leq x^{1, R} \leq 1$ and $x^{2, R} \geq x^{1, R}$.

Proof. Define $f\left(x^{L}, x^{1, R}, x^{2, R}, \delta\right)=\mathbb{P}[\eta$ hits $B(1, \delta)]$. We argue that $f$ is continuous. Suppose $\eta_{n}$ is a $\operatorname{SLE}_{\kappa}\left(\rho^{L} ; \rho^{1, R}, \rho^{2, R}\right)$ with force points $\left(x_{n}^{L} ; x_{n}^{1, R}\right.$, $\left.x_{n}^{2, R}\right)$ and assume $\left(x_{n}^{L}, x_{n}^{1, R}, x_{n}^{2, R}, \delta_{n}\right) \rightarrow\left(x^{L}, x^{1, R}, x^{2, R}, \delta\right)$. Denote by $g_{t}^{n}, W_{n}(t)$ the conformal map and the driving function for $\eta_{n}$. By Lemma A.4, we see that $W_{n}$ converges to $W$ in local uniform topology. Combining with [13], Section 4.7, we obtain that $g^{n}$ converges to $g$ in Carathéodory topology. This gives the continuity of $f$.

Define $p(\delta):=\sup f\left(x^{L}, x^{1, R}, x^{2, R}, \delta\right)$ where the supremum is taken over $x^{L} \leq 0, x^{1, R} \in[0,1], x^{2, R} \geq x^{1, R}$. Since (A.1) holds, for each $x^{L}, x^{1, R}, x^{2, R}$ we have $f\left(x^{L}, x^{1, R}, x^{2, R}, \delta\right) \rightarrow 0$ as $\delta \rightarrow 0$. When $\left|x^{L}\right|, x^{2, R} \rightarrow \infty$, the law of $\eta$ becomes the law of $\operatorname{SLE}_{\kappa}\left(\rho^{1, R}\right)$. This implies $p(\delta) \rightarrow 0$ as $\delta \rightarrow 0$.

Proof of Theorem A.1. Lemma A.3 gives the lower bound, and we only need to show the upper bound. Pick an integer $n$ such that $2^{n} \varepsilon \leq 1 / 4$. For $1 \leq k \leq$ $n$, let $T_{k}$ be the first time that $\eta$ hits the ball centered at 1 with radius $2^{n+1-k} \varepsilon(1-$ $\left.x^{R}\right)$. Define

$$
\mathcal{F}_{k}=\left\{\operatorname{Im} \eta\left(T_{k}\right) \geq \delta 2^{n+1-k} \varepsilon\left(1-x^{R}\right)\right\} .
$$

By Lemma A.3, we know that

$$
\begin{aligned}
\mathbb{P}\left[\tau_{\varepsilon} \leq S_{r}\right] & \leq \sum_{1}^{n} \mathbb{P}\left[\left\{T_{k}<\infty\right\} \cap \mathcal{F}_{k}\right]+\mathbb{P}\left[\left\{\tau_{\varepsilon} \leq S_{r}\right\} \bigcap_{1}^{n} \mathcal{F}_{k}^{c}\right] \\
& \lesssim 2^{n \alpha} \varepsilon^{\alpha}\left(1-x^{R}\right)^{\beta} \delta^{-\beta} r^{B}+\mathbb{P}\left[\left\{\tau_{\varepsilon} \leq S_{r}\right\} \bigcap_{1}^{n} \mathcal{F}_{k}^{c}\right]
\end{aligned}
$$


By a similar argument as in Lemma 4.4, there is a function $q(\delta) \rightarrow 0$ as $\delta \rightarrow 0$ which depends only on $\kappa, \rho^{L}, \rho^{1, R}$ and $\rho^{2, R}$ such that $\mathbb{P}\left[\bigcap_{1}^{n} \mathcal{F}_{k}^{c}\right] \leq q(\delta)^{n}$. Thus we have

$$
\mathbb{P}\left[\tau_{\varepsilon} \leq S_{r}\right] \lesssim 2^{n \alpha} \varepsilon^{\alpha}\left(1-x^{R}\right)^{\beta} \delta^{-\beta} r^{B}+q(\delta)^{n}
$$

This implies the conclusion.

Proof of COROllary A.2. Assume the same notation as in the proof of Theorem A.1. When $\rho^{L} \leq 0$, we have $B=0$, thus

$$
\mathbb{P}\left[\tau_{\varepsilon}<\infty\right] \lesssim 2^{n \alpha} \varepsilon^{\alpha}\left(1-x^{R}\right)^{\beta} \delta^{-\beta}+q(\delta)^{n} .
$$

This implies the conclusion.

Acknowledgments. The author acknowledges Hugo Duminil-Copin, Matan Harel, Aran Raoufi, Stanislav Smirnov and Vincent Tassion for helpful discussion on the Ising and FK-Ising model. The author thanks Gregory Lawler, David Wilson and Dapeng Zhan for helpful discussion on SLE estimates. The author appreciates the advice from two anonymous referees.

\section{REFERENCES}

[1] AhLfors, L. V. (2006). Lectures on Quasiconformal Mappings, 2nd ed. University Lecture Series 38. Amer. Math. Soc., Providence, RI. MR2241787

[2] Ahlfors, L. V. (2010). Conformal Invariants: Topics in Geometric Function Theory. AMS Chelsea Publishing, Providence, RI. Reprint of the 1973 original, With a foreword by Peter Duren, F. W. Gehring and Brad Osgood. MR2730573

[3] Alberts, T. and KozDron, M. J. (2008). Intersection probabilities for a chordal SLE path and a semicircle. Electron. Commun. Probab. 13 448-460. MR2430712

[4] Beffara, V. (2008). The dimension of the SLE curves. Ann. Probab. 36 1421-1452. MR2435854

[5] Benoist, S., Duminil-Copin, H. and Hongler, C. (2016). Conformal invariance of crossing probabilities for the Ising model with free boundary conditions. Ann. Inst. Henri Poincaré Probab. Stat. 52 1784-1798.

[6] Chelkak, D. (2016). Robust discrete complex analysis: A toolbox. Ann. Probab. 44 628-683. MR3456348

[7] Chelkak, D., Duminil-Copin, H. and Hongler, C. (2016). Crossing probabilities in topological rectangles for the critical planar FK-Ising model. Electron. J. Probab. 21 Paper No. 5, 28.

[8] Chelkak, D., Duminil-Copin, H., Hongler, C., Kemppainen, A. and Smirnov, S. (2014). Convergence of Ising interfaces to Schramm's SLE curves. C. R. Math. Acad. Sci. Paris 352 157-161.

[9] Chelkak, D. and Smirnov, S. (2012). Universality in the 2D Ising model and conformal invariance of fermionic observables. Invent. Math. 189 515-580. MR2957303

[10] Dubédat, J. (2009). Duality of Schramm-Loewner evolutions. Ann. Sci. Éc. Norm. Supér. (4) 42 697-724.

[11] Duplantier, B. (2004). Conformal fractal geometry and boundary quantum gravity. In Fractal Geometry and Applications: A Jubilee of Benoît Mandelbrot, Part 2 365-482. Amer. Math. Soc., Providence, RI. 
[12] Hongler, C. and KYTÖLÄ, K. (2013). Ising interfaces and free boundary conditions. J. Amer. Math. Soc. 26 1107-1189.

[13] Lawler, G. F. (2005). Conformally Invariant Processes in the Plane. Mathematical Surveys and Monographs 114. Amer. Math. Soc., Providence, RI.

[14] LAWLER, G. F. (2015). Minkowski content of the intersection of a Schramm-Loewner evolution (SLE) curve with the real line. J. Math. Soc. Japan 67 1631-1669.

[15] Lawler, G. F., Schramm, O. and Werner, W. (2001). Values of Brownian intersection exponents. I. Half-plane exponents. Acta Math. 187 237-273. MR1879850

[16] LaWler, G. F., Schramm, O. and Werner, W. (2002). One-arm exponent for critical 2D percolation. Electron. J. Probab. 7 no. 2, 13 pp. (electronic).

[17] Miller, J. and ShefFIELD, S. (2016). Imaginary geometry I: Interacting SLEs. Probab. Theory Related Fields 164 553-705.

[18] MilleR, J. and WU, H. (2017). Intersections of SLE Paths: The double and cut point dimension of SLE. Probab. Theory Related Fields 167 45-105.

[19] Pommerenke, C. (1992). Boundary Behaviour of Conformal Maps 299. Springer, Berlin.

[20] Rohde, S. and Schramm, O. (2005). Basic properties of SLE. Ann. of Math. (2) $161883-$ 924.

[21] Schramm, O. and Wilson, D. B. (2005). SLE coordinate changes. New York J. Math. 11 659-669 (electronic).

[22] Smirnov, S. and Werner, W. (2001). Critical exponents for two-dimensional percolation. Math. Res. Lett. 8 729-744.

[23] Viklund, F. J. and LaWler, G. F. (2012). Almost sure multifractal spectrum for the tip of an SLE curve. Acta Math. 209 265-322.

[24] Werner, W. and Wu, H. (2013). From CLE $(\kappa)$ to SLE $(\kappa, \rho)$. Electron. J. Probab. 18 1-20.

[25] WU, H. (2018). Polychromatic arm exponents for the critical planar FK-Ising model. J. Stat. Phys. 170 1177-1196.

[26] Wu, H. and ZHAN, D. (2017). Boundary arm exponents for SLE. Electron. J. Probab. 22 no. 89, 1-26. 